\title{
Towards a more holistic framework for solvent selection
}

\author{
Louis J. Diorazio,* David R. J. Hose, ${ }^{*}$ Neil K. Adlington \\ Pharmaceutical Development, AstraZeneca, Hulley Road, Macclesfield, Cheshire, \\ SK10 2NA, United Kingdom
}

\section{Supporting Information}

\section{Table of Contents}

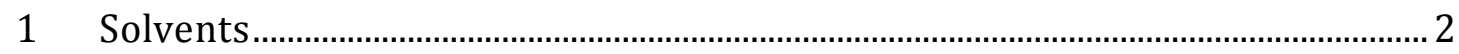

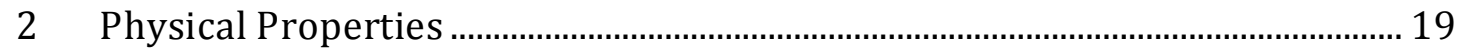

3 Physical Properties Summary Charts .......................................................................... 26

$4 \quad$ Computed Properties ...................................................................................................... 31

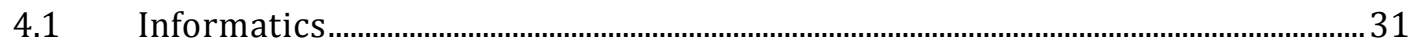

4.2 Quantum Mechanical (Gaussian). ………………......................................................... 31

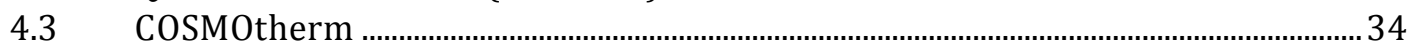

4.4 Chiral Molecules............................................................................................................. 36

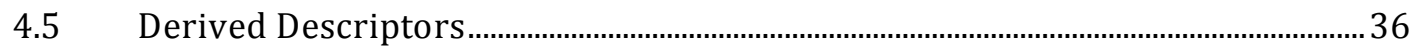

5 Calculated Properties Summary Charts .................................................................. 38

6 PLS Physical Properties versus Computed Descriptors: Predicted - Observed

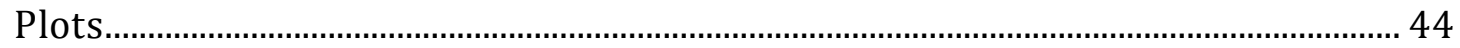




\section{SOLVENTS}

Table S1 lists all of the solvents in the database along with their corresponding CAS number, SMILES string and InChIKey.

\section{Table S1}

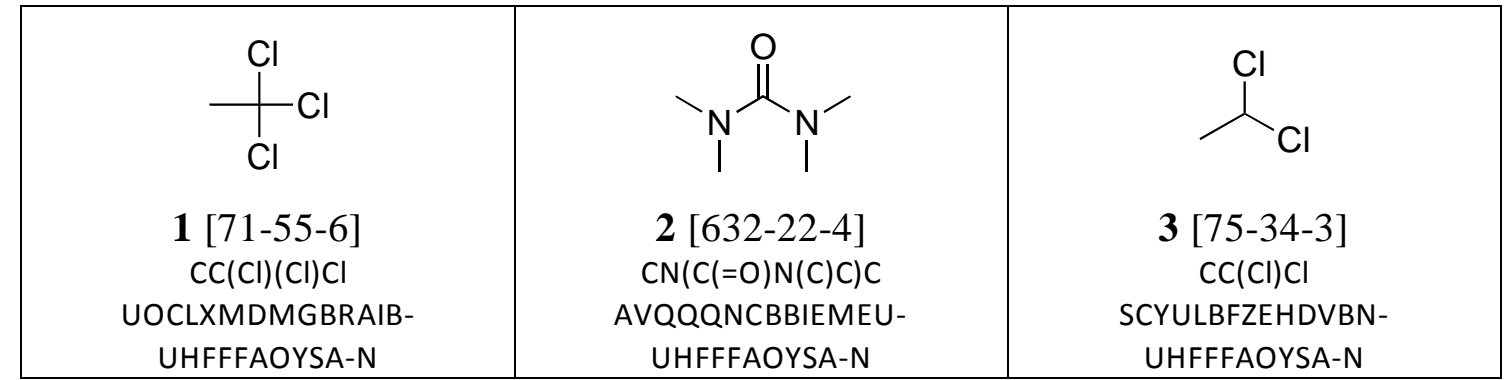

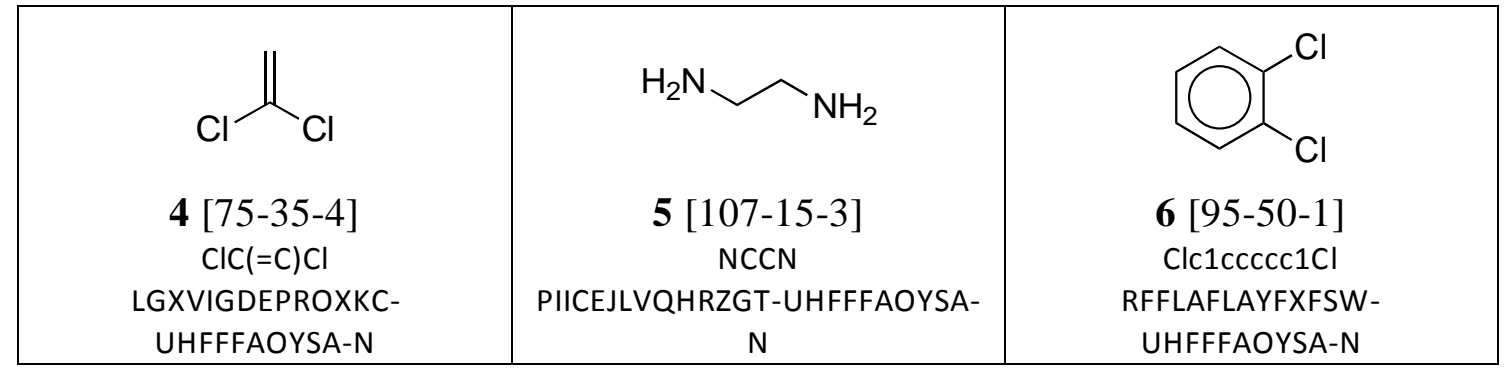

\begin{tabular}{|c|c|c|}
\hline & & \\
\hline 7 [107-06-2] & 8 [110-71-4] & 9 [107-21-1] \\
\hline $\begin{array}{c}\text { CICCCI } \\
\text { WSLDOOZREJYCGB- } \\
\text { UHFFFAOYSA-N }\end{array}$ & $\begin{array}{c}\text { COCCOC } \\
\text { XTHFKEDIFFGKHM- } \\
\text { UHFFFAOYSA-N }\end{array}$ & $\begin{array}{c}\text { OCCO } \\
\text { LYCAIKOWRPUZTN- } \\
\text { UHFFFAOYSA-N }\end{array}$ \\
\hline
\end{tabular}

\begin{tabular}{|c|c|c|}
\hline $\begin{array}{l}\mathbf{1 0}[108-67-8] \\
\text { Cc1cc(C)cc(c1)C } \\
\text { AUHZEENZYGFFBQ- } \\
\text { UHFFFAOYSA-N }\end{array}$ & $\begin{array}{c}11[106-42-3] \\
\text { Cc1ccc(cc1)C } \\
\text { URLKBWYHVLBVBO- } \\
\text { UHFFFAOYSA-N }\end{array}$ & $\begin{array}{c}12[123-91-1] \\
\text { O1CCOCC1 } \\
\text { RYHBNJHYFVUHQT- } \\
\text { UHFFFAOYSA-N }\end{array}$ \\
\hline $\begin{array}{c}13 \text { [872-50-4] } \\
\text { O=C1CCCN1C } \\
\text { SECXISVLQFMRJM- } \\
\text { UHFFFAOYSA-N }\end{array}$ & $\begin{array}{c}14[565-80-0] \\
\text { CC(C }(=0) C(C) C) C \\
\text { HXVNBWAKAOHACI- } \\
\text { UHFFFAOYSA-N }\end{array}$ & $\begin{array}{c}15[108-83-8] \\
\mathrm{CC}(\mathrm{CC}(=0) \mathrm{CC}(\mathrm{C}) \mathrm{C}) \mathrm{C} \\
\mathrm{N} \\
\text { PTTPXKJJBFFKCEK-UHFFFAOYSA- }\end{array}$ \\
\hline
\end{tabular}




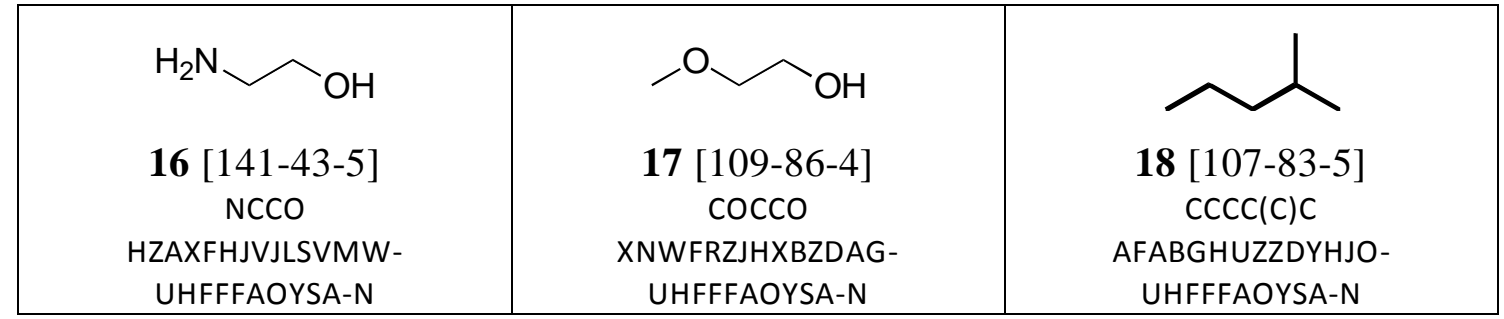

\begin{tabular}{|c|c|c|}
\hline & \\
$\mathbf{1 9}[96-47-9]$ & $\mathbf{2 0}[75-85-4]$ & $\mathbf{2 1}[78-83-1]$ \\
CC1CCCO1 & CCC(O)(C)C & OCC(C)C \\
JWUJQDFVADABEY- & MSXVEPNJUHWQHW- \\
UHFFFAOYSA-N & ZXEEKIIBDNHEJCQ-UHFFFAOYSA- \\
\hline
\end{tabular}

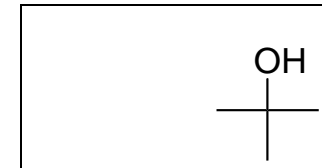

22 [75-65-0] $\mathrm{CC}(\mathrm{O})(\mathrm{C}) \mathrm{C}$ DKGAVHZHDRPRBMUHFFFAOYSA-N<smiles>CC(C)CCO</smiles>

25 [123-51-3] OCCC (C)C PHTQWCKDNZKARWUHFFFAOYSA-N<smiles>CC(=O)C(C)(C)C</smiles>

23 [75-97-8] $\mathrm{CC}(=0) \mathrm{C}(\mathrm{C})(\mathrm{C}) \mathrm{C}$ PJGSXYOJTGTZAVUHFFFAOYSA-N<smiles>CC(=O)CC(C)C</smiles>

26 [108-10-1] $\mathrm{CC}(\mathrm{CC}(=0) \mathrm{C}) \mathrm{C}$ NTIZESTWPVYFNLUHFFFAOYSA-N<smiles>CC(=O)C(C)C</smiles>

24 [563-80-4] $\mathrm{CC}(=0) \mathrm{C}(\mathrm{C}) \mathrm{C}$ SYBYTAAJFKOIEJ-UHFFFAOYSA$\mathrm{N}$<smiles>CC(=O)O</smiles>

\section{7 [64-19-7]}

$\mathrm{CC}(=0) 0$ QTBSBXVTEAMEQOUHFFFAOYSA-N

\begin{tabular}{|c|c|c|}
\hline $\mathrm{O}$ & 0 & $\mathrm{~N} \equiv$ \\
\hline $\mathbf{2 8}[108-24-7]$ & 29 [67-64-1] & $30[75-05-8]$ \\
\hline$C C(=0) O C(=0) C$ & $C C(=O) C$ & $\mathrm{CCHN}$ \\
\hline $\begin{array}{l}\text { WFDIJRYMOXRFFG- } \\
\text { UHFFFAOYSA-N }\end{array}$ & $\begin{array}{l}\text { CSCPPACGZOOCGX- } \\
\text { UHFFFAOYSA-N }\end{array}$ & $\begin{array}{c}\text { WEVYAHXRMPXWCK- } \\
\text { UHFFFAOYSA-N }\end{array}$ \\
\hline $\mathrm{O}$ & & \\
\hline 31 [98-86-2] & 32 [62-53-3] & 33 [100-66-3] \\
\hline$C C(=0) \operatorname{cocccc} 1$ & Nc1ccccc1 & $\operatorname{coc} 1 \operatorname{ccccc} 1$ \\
\hline $\begin{array}{l}\text { KWOLFJPFCHCOCG- } \\
\text { UHFFFAOYSA-N }\end{array}$ & $\begin{array}{l}\text { PAYRUJLWNCNPSJ- } \\
\text { UHFFFAOYSA-N }\end{array}$ & $\begin{array}{l}\text { RDOXTESZEPMUJZ- } \\
\text { UHFFFAOYSA-N }\end{array}$ \\
\hline
\end{tabular}




\begin{tabular}{|c|c|c|}
\hline $\begin{array}{c}34 \text { [71-43-2] } \\
\text { c1ccccc1 } \\
\text { UHOVQNZJYSORNB- } \\
\text { UHFFFAOYSA-N }\end{array}$ & $\begin{array}{l}35[100-47-0] \\
\text { N\#Cc1ccccc1 } \\
\text { JFDZBHWFFUWGJE- } \\
\text { UHFFFAOYSA-N }\end{array}$ & $\begin{array}{l}36 \text { [98-08-8] } \\
\text { FC(c1ccccc1)(F)F } \\
\text { GETTZEONDQJALK- } \\
\text { UHFFFAOYSA-N }\end{array}$ \\
\hline $\begin{array}{l}\text { OCH } \\
\text { OCc1cccc1 } \\
\text { WVDDGKGOMKODPV- } \\
\text { UHFFFAOYSA-N }\end{array}$ & $\begin{array}{l}38 \text { [108-86-1] } \\
\text { Brc1cccc1 } 1 \\
\text { QARVLSVVCXYDNA- } \\
\text { UHFFFAOYSA-N }\end{array}$ & $\begin{array}{l}39 \text { [71-36-3] } \\
\text { CCCCO } \\
\text { LRHPLDYGYMQRHN- } \\
\text { UHFFFAOYSA-N }\end{array}$ \\
\hline
\end{tabular}

\begin{tabular}{|c|c|c|}
\hline $\begin{array}{c}\mathbf{4 0 H}[78-92-2] \\
\text { CCC(O)C }\end{array}$ & $\begin{array}{c}\mathbf{4 1}[78-93-3] \\
\text { CCC(=O)C }\end{array}$ \\
$\begin{array}{c}\text { BTANRVKWQNVYAZ- } \\
\text { UHFFFAOYSA-N }\end{array}$ & $\begin{array}{c}\mathbf{4 2}[109-74-0] \\
\text { CCCC\#N } \\
\text { ZWEHNKRNPOVVGH- } \\
\text { UHFFFAOYSA-N }\end{array}$ \\
UHRLNFWIYMESJ- \\
UHFFFAOYSA-N
\end{tabular}

\begin{tabular}{|c|c|c|}
\hline $\mathrm{S}=\mathrm{C}=\mathrm{S}$ & & Il \\
\hline $43[75-15-0]$ & 44 [108-90-7] & 45 [67-66-3] \\
\hline $\begin{array}{l}\text { QGJOPFRUJISHPQ- } \\
\text { UHFFFAOYSA-N }\end{array}$ & $\begin{array}{c}\text { MVPPADPHJFYWMZ- } \\
\text { UHFFFAOYSA-N }\end{array}$ & $\begin{array}{c}\text { HEDRZPFGACZZDS- } \\
\text { UHFFFAOYSA-N }\end{array}$ \\
\hline
\end{tabular}

\begin{tabular}{|c|c|c|}
\hline $\begin{array}{c}\text { C1CCC2C(C1)CCCC2 } \\
\text { NNBZCPXTIHJBJ-UHFFFAOYSA- } \\
\mathrm{N}\end{array}$ & $\begin{array}{c}\text { C1CCCCC1 } \\
\text { XDTMQSROBMDMFD- } \\
\text { UHFFFAOYSA-N }\end{array}$ & $\begin{array}{c}\mathbf{4 8}[108-93-0] \\
\text { OC1CCCCC1 } \\
\text { HPXRVTGHNJAIIH- } \\
\text { UHFFFAOYSA-N }\end{array}$ \\
\hline
\end{tabular}

\begin{tabular}{|c|c|c|}
\hline & & \\
\hline 49 [108-94-1] & 50 [5614-37-9] & 51 [124-18-5] \\
\hline $\mathrm{O}=\mathrm{C} 1 \mathrm{CCCCC} 1$ & COC1CCCC1 & сссссссссс \\
\hline $\begin{array}{l}\text { JHIVVAPYMSGYDF- } \\
\text { UHFFFAOYSA-N }\end{array}$ & $\begin{array}{c}\text { SKTCDJAMAYNROS- } \\
\text { UHFFFAOYSA-N }\end{array}$ & $\begin{array}{l}\text { DIOQZVSQGTUSAI- } \\
\text { UHFFFAOYSA-N }\end{array}$ \\
\hline
\end{tabular}




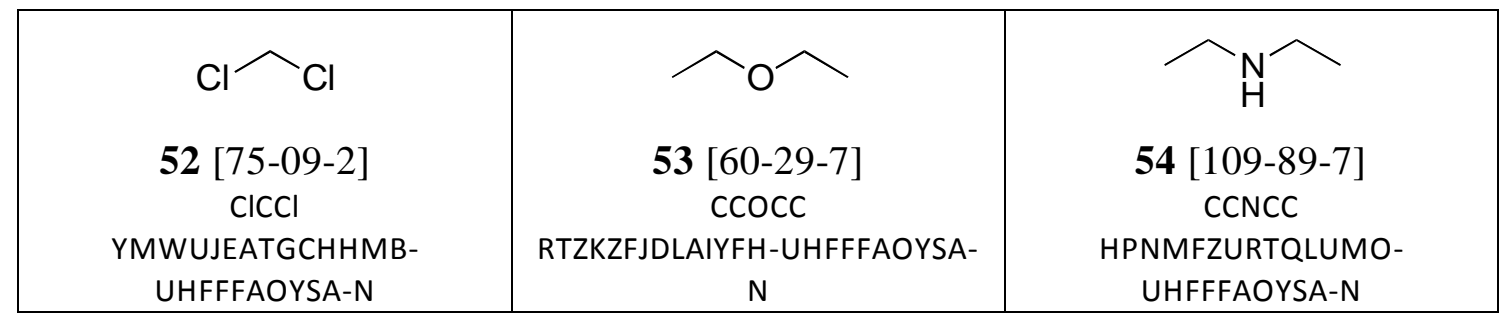

\begin{tabular}{|c|c|c|}
\hline $\begin{array}{c}\text { CCOC(=O)OCC } \\
\text { OIFBSDVPJOWBCH- } \\
\text { UHFFFAOYSA-N }\end{array}$ & $\begin{array}{c}\mathbf{5 6}[111-46-6] \\
\text { OCCOCCO } \\
\text { MTHSVFCYNBDYFN- } \\
\text { UHFFFAOYSA-N }\end{array}$ & $\begin{array}{c}\mathbf{5} \text { CCOCCOCCOCC } \\
\text { RRQYJINTUHWNHW- } \\
\text { UHFFFAOYSA-N }\end{array}$ \\
\hline
\end{tabular}

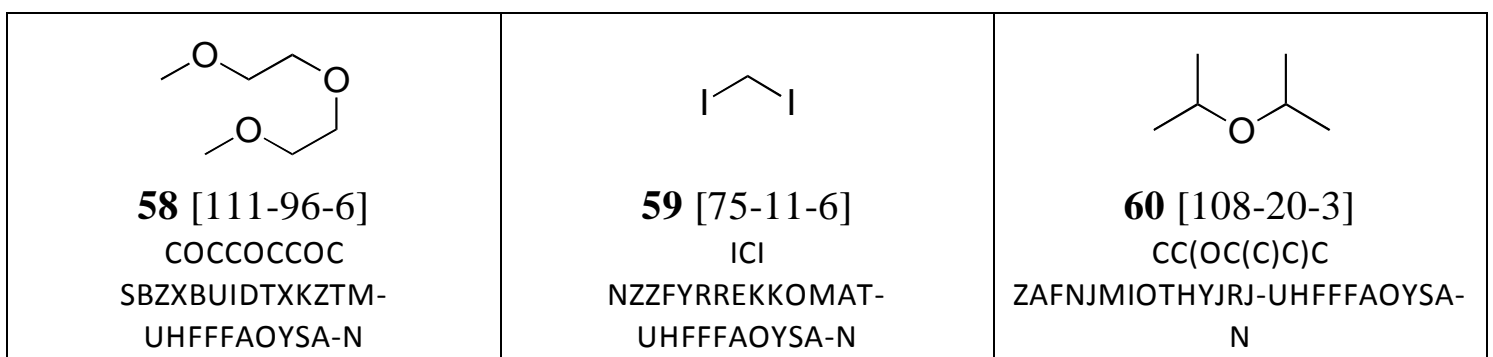

\begin{tabular}{|c|c|c|}
\hline & \\
$\mathbf{6 1}[67-68-5]$ & \\
CS(=O)C \\
$\begin{array}{c}\text { IAZDPXIOMUYVGZ- } \\
\text { UHFFFAOYSA-N }\end{array}$ & $\begin{array}{c}\mathbf{6 3}[111-43-3] \\
\text { CURCOCCC } \\
\text { CUPTKYDGMDSBL- } \\
\text { UHFFFAOYSA-N }\end{array}$ \\
\hline
\end{tabular}

\begin{tabular}{|c|c|c|}
\hline $\begin{array}{l}\mathbf{6 4}[101-84-8] \\
\text { c1ccc(cc1)Oc1cccc1 } \\
\text { USIUVYZYUHIAEV- } \\
\text { UHFFFAOYSA-N }\end{array}$ & $\begin{array}{c}\mathbf{6 5}[64-17-5] \\
\text { CCO } \\
\text { LFQSCWFLJHTTHZ- } \\
\text { UHFFFAOYSA-N }\end{array}$ & $\begin{array}{l}66[141-78-6] \\
\text { CCOC(=O)C } \\
\text { XEKOWRVHYACXOJ- } \\
\text { UHFFFAOYSA-N }\end{array}$ \\
\hline $\begin{array}{c}67[103-73-1] \\
\text { CCOc1cccc1 } \\
\text { DLRJIFUOBPOJNS-UHFFFAOYSA- } \\
\mathrm{N}\end{array}$ & $\begin{array}{l}\mathbf{6 8} \text { [462-06-6] } \\
\text { Fc1ccccc1 } \\
\text { PYLWMHQQBFSUBP- } \\
\text { UHFFFAOYSA-N }\end{array}$ & $\begin{array}{c}\mathrm{H}_{2} \mathrm{~N} \mathrm{O} \\
69[75-12-7] \\
\text { NC=O } \\
\text { ZHNUHDYFZUAESO- } \\
\text { UHFFFAOYSA-N }\end{array}$ \\
\hline
\end{tabular}




\begin{tabular}{|c|c|c|}
\hline $\mathrm{HO} \approx \mathrm{O}$ & $\mathrm{H}$ & ${ }_{/}$ \\
\hline 70 [64-18-6] & $71[56-81-5]$ & 72 [680-31-9] \\
\hline $\mathrm{OC}=\mathrm{O}$ & $\mathrm{OCC}(\mathrm{CO}) \mathrm{O}$ & $\mathrm{CN}(\mathrm{P}(=\mathrm{O})(\mathrm{N}(\mathrm{C}) \mathrm{C}) \mathrm{N}(\mathrm{C}) \mathrm{C}) \mathrm{C}$ \\
\hline $\begin{array}{l}\text { BDAGIHXWWSANSR- } \\
\text { UHFFFAOYSA-N }\end{array}$ & $\begin{array}{c}\text { PEDCQBHIVMGVHV- } \\
\text { UHFFFAOYSA-N }\end{array}$ & $\begin{array}{l}\text { GNOIPBMMFNIUFM- } \\
\text { UHFFFAOYSA-N }\end{array}$ \\
\hline
\end{tabular}

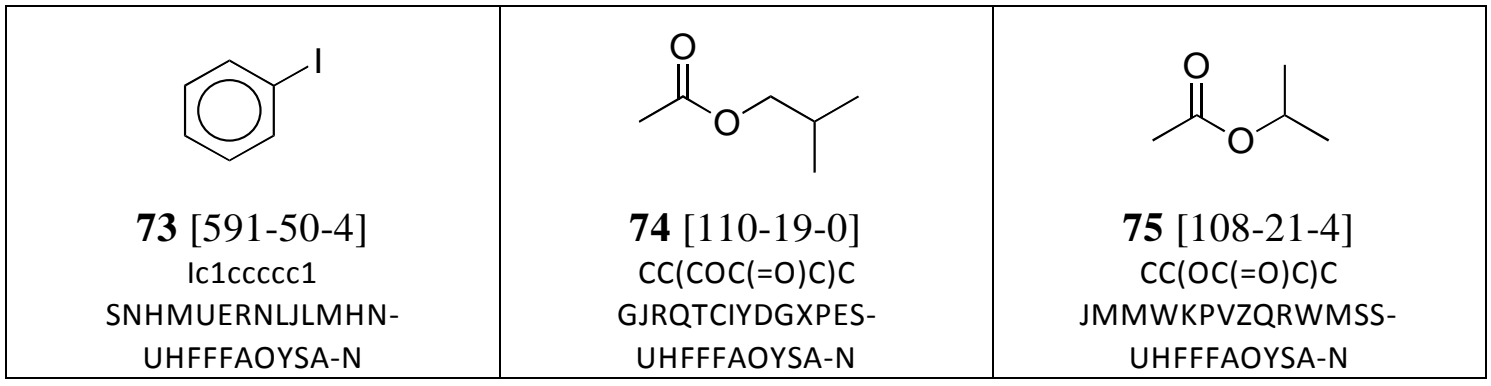

\begin{tabular}{|c|c|c|}
\hline$-\mathrm{OH}$ & & \\
\hline 76 [67-56-1] & 77 [79-20-9] & 78 [110-91-8] \\
\hline $\mathrm{CO}$ & $\operatorname{coc}(=0) C$ & C1CNCCO1 \\
\hline $\begin{array}{c}\text { OKKJLVBELUTLKV-UHFFFAOYSA- } \\
\mathrm{N}\end{array}$ & $\begin{array}{l}\text { KXKVLQRXCPHEJC- } \\
\text { UHFFFAOYSA-N }\end{array}$ & $\begin{array}{l}\text { YNAVUWVOSKDBBP- } \\
\text { UHFFFAOYSA-N }\end{array}$ \\
\hline
\end{tabular}

\begin{tabular}{|c|c|c|}
\hline $\begin{array}{c}\mid \\
79[127-19-5] \\
\text { CC(=O)N(C)C } \\
\text { FXHOOIRPVKKKFG- } \\
\text { UHFFFAOYSA-N }\end{array}$ & $\begin{array}{c}\mathrm{O}_{\approx}[68-12-2] \\
\mathrm{O}=\mathrm{CN}(\mathrm{C}) \mathrm{C} \\
\text { ZMXDDKWLCZADIW- } \\
\text { UHFFFAOYSA-N }\end{array}$ & $\begin{array}{c}\mathbf{8 1}[123-86-4] \\
\text { CCCCOC }(=0) C \\
\text { DKPFZGUDAPQIHT- } \\
\text { UHFFFAOYSA-N }\end{array}$ \\
\hline
\end{tabular}

\begin{tabular}{|c|c|c|}
\hline $\begin{array}{c}\text { CCCCCCCC } \\
\text { IMNFUFMRHMDMM- } \\
\text { UHFFFAOYSA-N }\end{array}$ & $\begin{array}{c}\mathbf{8 3}[110-54-3] \\
\text { CCCCCCC } \\
\text { VLKZOEOYAKHREP- } \\
\text { UHFFFAOYSA-N }\end{array}$ & $\begin{array}{c}\mathbf{8 4}[98-95-3] \\
\text { O=[N+](c1)cccc1)[O- }] \\
\text { LQNUZADURLCDLV- } \\
\text { UHFFFAOYSA-N }\end{array}$ \\
\hline
\end{tabular}




\begin{tabular}{|c|c|c|}
\hline $\begin{array}{c}\mathrm{O}^{-} \\
1 \\
\mathrm{~N}_{+} \\
+\end{array}$ & & $\mathrm{N}^{-}$ \\
\hline 85 [75-52-5] & 86 [79-16-3] & 87 [123-39-7] \\
\hline $\mathrm{C}[\mathrm{N}+]([\mathrm{O}-])=0$ & $C N C(=0) C$ & $C N C=0$ \\
\hline $\begin{array}{l}\text { LYGJENNIWJXYER- } \\
\text { UHFFFAOYSA-N }\end{array}$ & $\begin{array}{l}\text { OHLUUHNLEMFGTQ- } \\
\text { UHFFFAOYSA-N }\end{array}$ & $\begin{array}{c}\text { ATHHXGZTWNVVOU- } \\
\text { UHFFFAOYSA-N }\end{array}$ \\
\hline
\end{tabular}

\begin{tabular}{|c|c|}
\hline $\begin{array}{c}\text { Be }[111-84-2] \\
\text { CCCCCCCCC } \\
\text { BKIMMITUMNOMOS- } \\
\text { UHFFFAOYSA-N }\end{array}$ & $\begin{array}{c}\mathbf{9 0}[109-66-0] \\
\text { CCCCC } \\
\text { TVMXDCGIABBOFY- } \\
\text { UHFFFAOYSA-N }\end{array}$ \\
$\begin{array}{c}\text { OFBQJSOFODEBGM- } \\
\text { UHFFFAOYSA-N }\end{array}$ \\
\hline
\end{tabular}

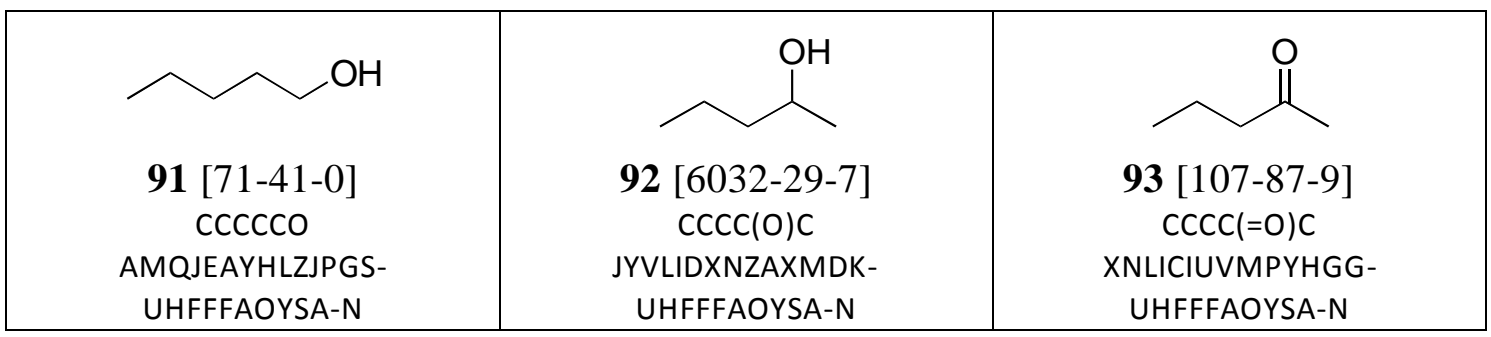

\begin{tabular}{|c|c|c|}
\hline & & \\
\hline $\begin{array}{c}94[584-02-1] \\
\text { CCC(CC)O } \\
\text { AQIXEPGDORPWBJ- } \\
\text { UHFFFAOYSA-N }\end{array}$ & $\begin{array}{c}95[96-22-0] \\
C C C(=0) C C \\
\text { FDPIMTJIUBPUKL-UHFFFAOYSA- } \\
N\end{array}$ & $\begin{array}{c}96[110-89-4] \\
\text { C1CCCNC1 } \\
\text { NQRYJNQNLNOLGT- } \\
\text { UHFFFAOYSA-N }\end{array}$ \\
\hline
\end{tabular}

\begin{tabular}{|c|c|c|}
\hline $\begin{array}{l}97[71-23-8] \\
\text { CCCO } \\
\text { BDERNNFJNOPAEC- } \\
\text { UHFFFAOYSA-N }\end{array}$ & $\begin{array}{l}98[67-63-0] \\
\mathrm{CC}(0) \mathrm{O} \\
\text { KFZMGEQAYNKOFK- } \\
\text { UHFFFAOYSA-N }\end{array}$ & $\begin{array}{c}99 \text { OH } \\
\text { CCC } 79-09-4] \\
\text { XBDQKXXYIPTUBI- } \\
\text { UHFFFAOYSA-N }\end{array}$ \\
\hline $\begin{array}{c}\text { N } \\
100[107-12-0] \\
\text { CCC\#N } \\
\text { FVSKHRXBFJPNKK- } \\
\text { UHFFFAOYSA-N }\end{array}$ & $\begin{array}{c}101 \text { [110-86-1] } \\
\text { c1cccnc1 } \\
\text { JUJWROOIHBZHMG- } \\
\text { UHFFFAOYSA-N }\end{array}$ & $\begin{array}{l}\mathbf{1 0 2}[91-22-5] \\
\text { c1ccc2c(c1)nccc2 } \\
\text { SMWDFEZZVXVKRB- } \\
\text { UHFFFAOYSA-N }\end{array}$ \\
\hline
\end{tabular}




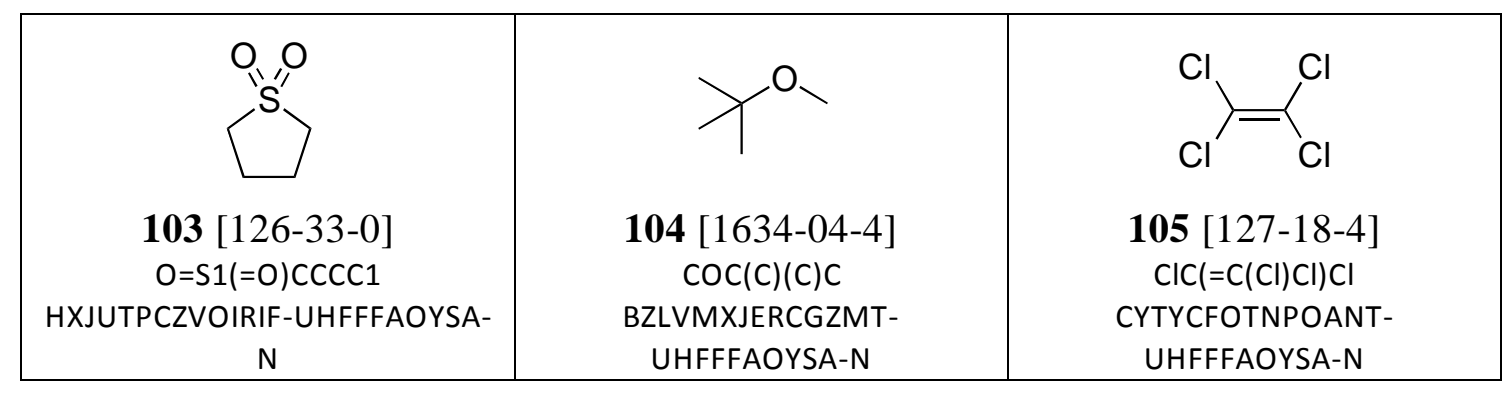

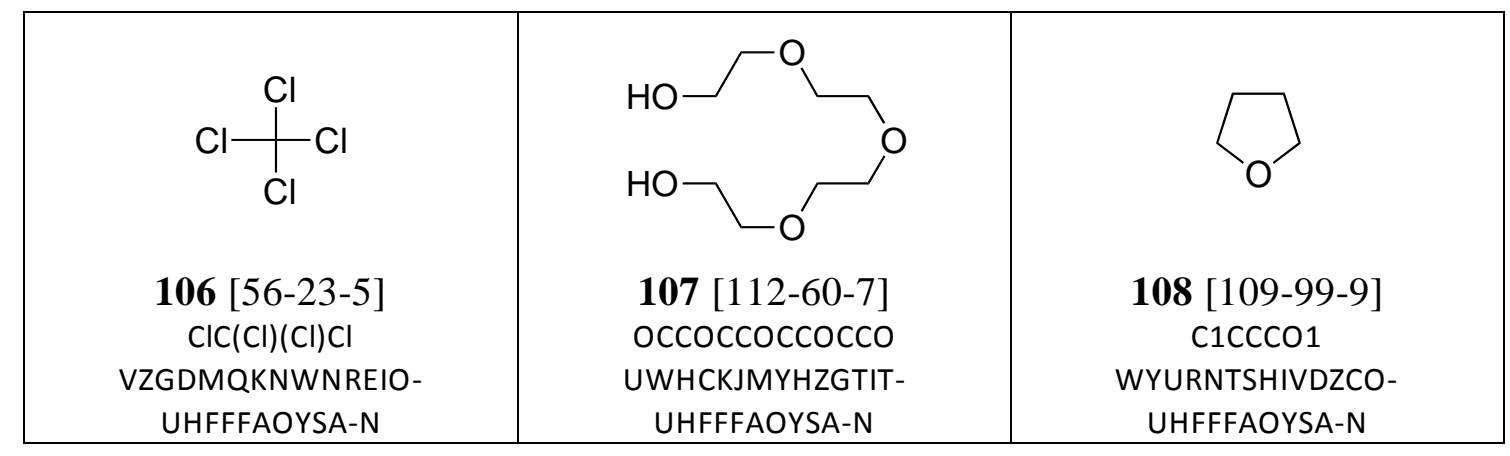

\begin{tabular}{|c|c|c|}
\hline $\begin{array}{c}109[119-64-2] \\
\text { C1CCC2C(C1)ccc2 } \\
\text { CXWXOJXEFPUFDZ- } \\
\text { UHFFFAOYSA-N }\end{array}$ & $\begin{array}{c}110[108-88-3] \\
\text { CCICCCC1 } \\
\text { YXFVVABEGXRONW- } \\
\text { UHFFFAOYSA-N }\end{array}$ \\
$\begin{array}{c}\text { CCCCN(CCCC)CCCC } \\
\text { IMFACGCPASFAPR- } \\
\text { UHFFFAOYSA-N }\end{array}$ \\
\hline
\end{tabular}

\begin{tabular}{|c|c|c|}
\hline $112[79-01-6]$ \\
$\begin{array}{c}C I C=C(C I) C l \\
\text { XSTXAVWGXDQKEL- } \\
\text { UHFFFAOYSA-N }\end{array}$ & $\begin{array}{c}113[121-44-8] \\
\text { CCN(CC)CC } \\
\text { ZMANZCXOSIPKH- } \\
\text { UHFFFAOYSA-N }\end{array}$ \\
\hline
\end{tabular}

\begin{tabular}{|c|c|c|}
\hline & $\mathrm{H}_{2} \mathrm{O}$ & \\
\hline 115 [112-49-2] & 116 [7732-18-5] & 117 [121-69-7] \\
\hline coccoccoccoc & 0 & $\mathrm{CN}(\operatorname{c} 1 \operatorname{ccccc} 1) \mathrm{C}$ \\
\hline $\begin{array}{l}\text { YFNKIDBQEZZDLK- } \\
\text { UHFFFAOYSA-N }\end{array}$ & $\begin{array}{l}\text { XLYOFNOQVPJJNP- } \\
\text { UHFFFAOYSA-N }\end{array}$ & $\begin{array}{l}\text { JLTDJTHDQAWBAV- } \\
\text { UHFFFAOYSA-N }\end{array}$ \\
\hline
\end{tabular}




\begin{tabular}{|c|c|c|}
\hline $\begin{array}{c}118 \text { [108-87-2] } \\
\text { CC1CCCCC1 } \\
\text { UAEPNZWRGJTJPN- } \\
\text { UHFFFAOYSA-N }\end{array}$ & $\begin{array}{l}\text { O=C1N(C)CCCN1C } \\
\text { GUVUOGQBMYCBQP- } \\
\text { UHFFFAOYSA-N }\end{array}$ & $\begin{array}{l}\mathbf{1 2 0}[80-73-9] \\
\text { CN1CCN(C1=O)C } \\
\text { CYSGHNMQYZDMIA- } \\
\text { UHFFFAOYSA-N }\end{array}$ \\
\hline $\begin{array}{l}\text { Z21 [2687-91-4] } \\
\text { CCN1CCCC1=O } \\
\text { UHFAREAONNGOYSA-N }\end{array}$ & $\begin{array}{l}122[994-05-8] \\
\text { CCC(OC)(C)C } \\
\text { HVZJRWJGKQPSFL- } \\
\text { UHFFFAOYSA-N }\end{array}$ & $\begin{array}{c}123 \text { [646-06-0] } \\
\text { C1OCCO1 } \\
\text { WNXJIVFYUVYPPR- } \\
\text { UHFFFAOYSA-N }\end{array}$ \\
\hline $\begin{array}{l}\mathrm{OH} \\
\mathbf{1 2 4}[97-64-3] \\
\text { CCOC(=O)C(O)C } \\
\text { LZCLXQDLBQLTDK- } \\
\text { UHFFFAOYSA-N }\end{array}$ & $\begin{array}{c}125[7778-85-0] \\
\text { COCC(OC)C } \\
\text { LEEANUDEDHYDTG- } \\
\text { UHFFFAOYSA-N }\end{array}$ & $\begin{array}{l}126 \text { [107-98-2] } \\
\text { COCC(O)C } \\
\text { ARXJGSRGQADJSQ- } \\
\text { UHFFFAOYSA-N }\end{array}$ \\
\hline
\end{tabular}

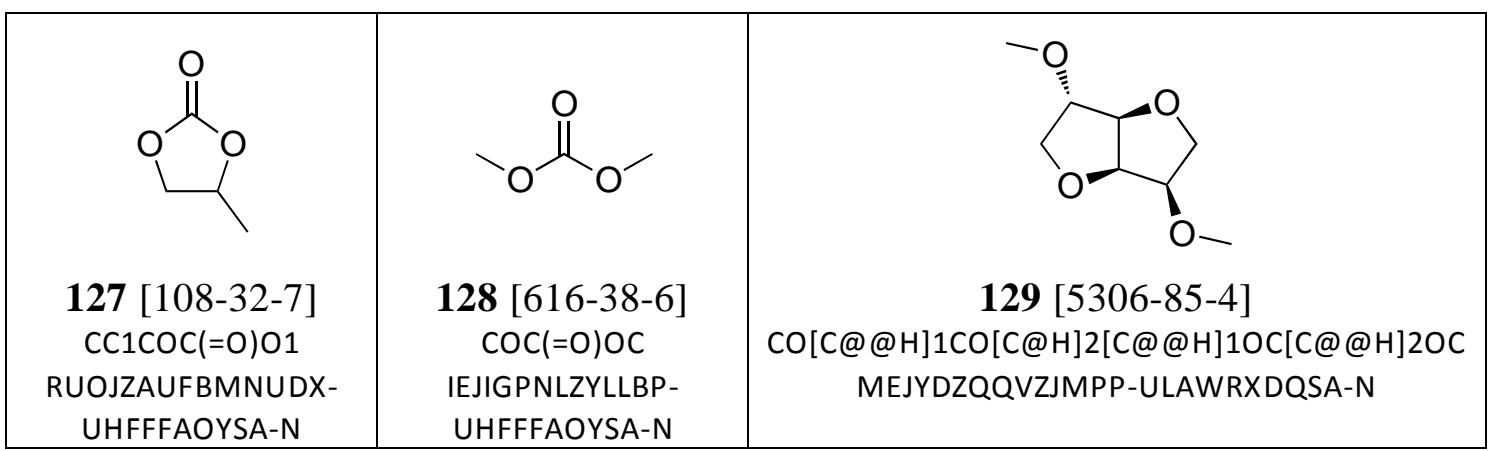<smiles>COC(OC)(OC)OC</smiles>

130 [1850-14-2] $\mathrm{COC}(\mathrm{OC})(\mathrm{OC}) \mathrm{OC}$ AHJWSRRHTXRLAQUHFFFAOYSA-N<smiles>OCC(F)(F)F</smiles>

131 [75-89-8] OCC $(F)(F) F$ RHQDFWAXVIIEBNUHFFFAOYSA-N<smiles>CC1=CC(=O)CC(C)(C)C1</smiles>

132 [78-59-1] $C C 1=C C(=0) C C(C 1)(C) C$ HJOVHMDZYOCNQWUHFFFAOYSA-N 


\begin{tabular}{|c|c|c|}
\hline $\begin{array}{l}133 \text { [583-60-8] } \\
\text { CC1CCCCC } 1=0 \\
\text { LFSAPCRASZRSKS-UHFFFAOYSA- } \\
N\end{array}$ & $\begin{array}{l}\mathbf{1 3 4}[111-15-9] \\
\text { CCOCCOC }(=0) C \\
\text { SVONRAPFPVNKG- } \\
\text { UHFFFAOYSA-N }\end{array}$ & $\begin{array}{c}135[141-97-9] \\
\text { CCOC }(=0) C C(=0) C \\
\text { XYIBRDXRRQCHLP- } \\
\text { UHFFFAOYSA-N }\end{array}$ \\
\hline $\begin{array}{l}\mathbf{1 3 6}[109-06-8] \\
\text { Cc1ccccn1 } \\
\begin{array}{c}\text { BSKHPKMHTQYZBB- } \\
\text { UHFFFAOYSA-N }\end{array}\end{array}$ & $\begin{array}{l}\text { 137 [108-48-5] } \\
\text { Cc1cccc(n1)C } \\
\text { OISVCGZHLKNMSJ- } \\
\text { UHFFFAOYSA-N }\end{array}$ & $\begin{array}{c}138[102-71-6] \\
\text { OCCN(CCO)CCO } \\
\text { GSEJCLTVZPLZKY-UHFFFAOYSA- } \\
\mathrm{N}\end{array}$ \\
\hline $\begin{array}{c}139[4829-04-3] \\
\text { C1SCCS1 } \\
\text { IMLSAISZLJGWPP-UHFFFAOYSA- } \\
\text { N }\end{array}$ & $\begin{array}{c}140[616-42-2] \\
\text { COS(=O)OC } \\
\text { BDUPRNVPXOHWIL- } \\
\text { UHFFFAOYSA-N }\end{array}$ & 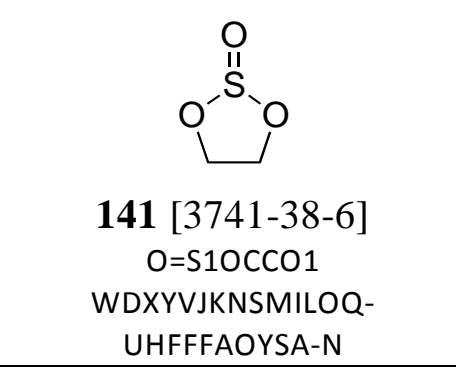 \\
\hline
\end{tabular}

\begin{tabular}{|c|c|c|}
\hline & & \\
\hline 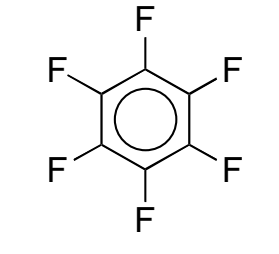 & r & $\mathrm{F}_{F}$ \\
\hline $142[392-56-3]$ & 143 [434-64-0] & $144[60433-11-6]$ \\
\hline $\begin{array}{l}F c 1 c(F) c(F) c(c(c 1 F) \\
F) F\end{array}$ & $\begin{array}{l}F c 1 c(F) c(F) c(c(c 1 F) C(F)( \\
F) F) F\end{array}$ & $\begin{array}{c}\mathrm{FC} 12 \mathrm{C}(\mathrm{F})(\mathrm{C}(\mathrm{F})(\mathrm{F}) \mathrm{C}(\mathrm{C}(\mathrm{C} 2(\mathrm{~F}) \mathrm{F})(\mathrm{F}) \mathrm{F})(\mathrm{F}) \mathrm{F}) \mathrm{C}(\mathrm{F})(\mathrm{F}) \mathrm{C}(\mathrm{C}(\mathrm{C} 1(\mathrm{~F}) \mathrm{F} \\
)(\mathrm{F}) \mathrm{F})(\mathrm{F}) \mathrm{F}\end{array}$ \\
\hline $\begin{array}{c}\text { ZQBFAOFFOQMS } \\
\text { GJ-UHFFFAOYSA- } \\
\mathrm{N}\end{array}$ & $\begin{array}{l}\text { USPWUOFNOTUBAD- } \\
\text { UHFFFAOYSA-N }\end{array}$ & UWEYRJFJVCLAGH-UHFFFAOYSA-N \\
\hline
\end{tabular}

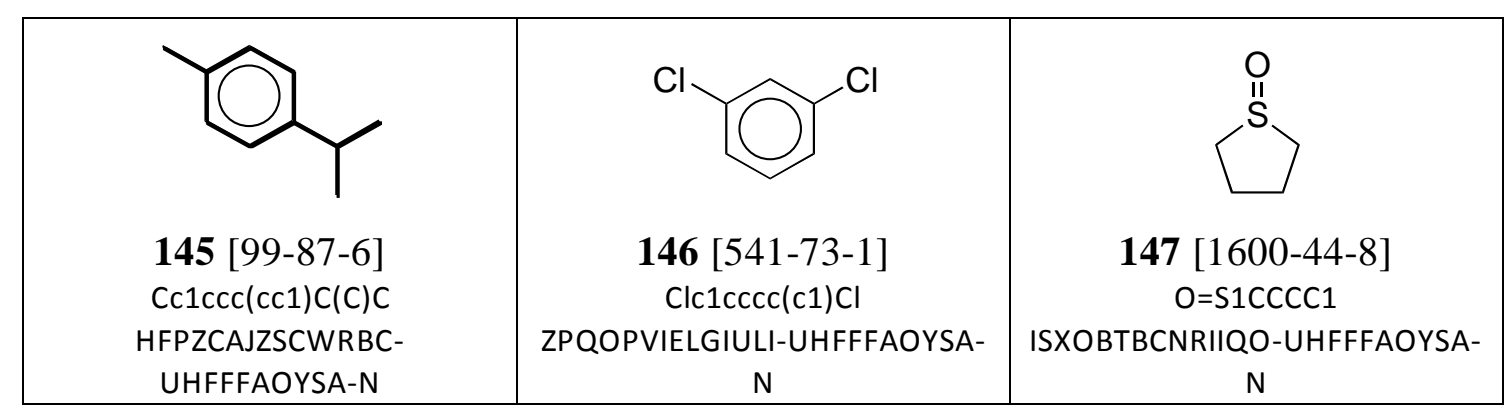




\begin{tabular}{|c|c|c|}
\hline &
\end{tabular}<smiles>CN1CCCCCC1=O</smiles>

151 [2556-73-2]

CN1CCCCCC $1=0$ ZWXPDGCFMMFNRWUHFFFAOYSA-N<smiles>CCN(CC)S(=O)(=O)N(CC)CC</smiles>

152 [2832-49-7]

$\operatorname{CCN}(\mathrm{S}(=0)(=0) \mathrm{N}(\mathrm{CC}) \mathrm{CC}) \mathrm{CC}$ IYIAWAACGTUPCCUHFFFAOYSA-N<smiles>CN(C)P(=O)(N(C)C)N(C)C</smiles>

153 [2511-17-3] $\mathrm{CN}(\mathrm{P}(=\mathrm{O})(\mathrm{N}(\mathrm{C}) \mathrm{C}) \mathrm{C}) \mathrm{C}$ YRWJRJCUZYGLPNUHFFFAOYSA-N<smiles>COC(C)(C)OC</smiles>

154 [77-76-9] $\operatorname{COC}(\mathrm{OC})(\mathrm{C}) \mathrm{C}$ HEWZVZIVELJPQZUHFFFAOYSA-N<smiles>CC1(C)OCC(CO)O1</smiles>

155 [100-79-8] OCC1COC(O1)(C)C RNVYQYLELCKWANUHFFFAOYSA-N

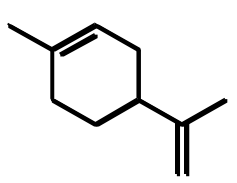

156 [5989-27-5] $\mathrm{CC} 1=\mathrm{CCC}(\mathrm{CC} 1) \mathrm{C}(=\mathrm{C}) \mathrm{C}$ XMGQYMWWDOXHJMUHFFFAOYSA-N

\begin{tabular}{|c|c|c|}
\hline 157 [108-29-2] & $158[6674-22-2]$ & 159 [7087-68-5] \\
\hline $\operatorname{cc} 1 \mathrm{CCC}(=0) 01$ & $\mathrm{C} 1 \mathrm{CCN} 2 \mathrm{C}(=\mathrm{NCCC} 2) \mathrm{CC} 1$ & $\operatorname{CCN}(C(C) C) C(C) C$ \\
\hline $\begin{array}{l}\text { GAEKPEKOJKCEMS- } \\
\text { UHFFFAOYSA-N }\end{array}$ & $\begin{array}{c}\text { GQHTUMJGOHRCHB- } \\
\text { UHFFFAOYSA-N }\end{array}$ & $\begin{array}{c}\text { JGFZNNIVVJXRND- } \\
\text { UHFFFAOYSA-N }\end{array}$ \\
\hline & & \\
\hline 160 [156-59-2] & $161[156-60-5]$ & $162[110-80-5]$ \\
\hline $\mathrm{Cl} / \mathrm{C}=\mathrm{C} / \mathrm{Cl}$ & $\mathrm{Cl} / \mathrm{C}=\mathrm{C} \backslash \mathrm{Cl}$ & occocc \\
\hline $\begin{array}{c}\text { KFUSEUYYWQURPO- } \\
\text { OWOJBTEDSA-N }\end{array}$ & $\begin{array}{c}\text { KFUSEUYYWQURPO- } \\
\text { UPHRSURJSA-N }\end{array}$ & $\begin{array}{c}\text { ZNQVEEAIQZEUHB- } \\
\text { UHFFFAOYSA-N }\end{array}$ \\
\hline & & \\
\hline 163 [591-78-6] & 164 [107-31-3] & 165 [109-94-4] \\
\hline $\operatorname{ccccc}(=0) c$ & & $\mathrm{CCOC}=\mathrm{O}$ \\
\hline $\begin{array}{l}\text { QQZOPKMRPOGIEB- } \\
\text { UHFFFAOYSA-N }\end{array}$ & $\begin{array}{l}\text { TZIHFWKZFHZASV- } \\
\text { UHFFFAOYSA-N }\end{array}$ & $\begin{array}{c}\text { WBJINCZRORDGAQ- } \\
\text { UHFFFAOYSA-N }\end{array}$ \\
\hline
\end{tabular}



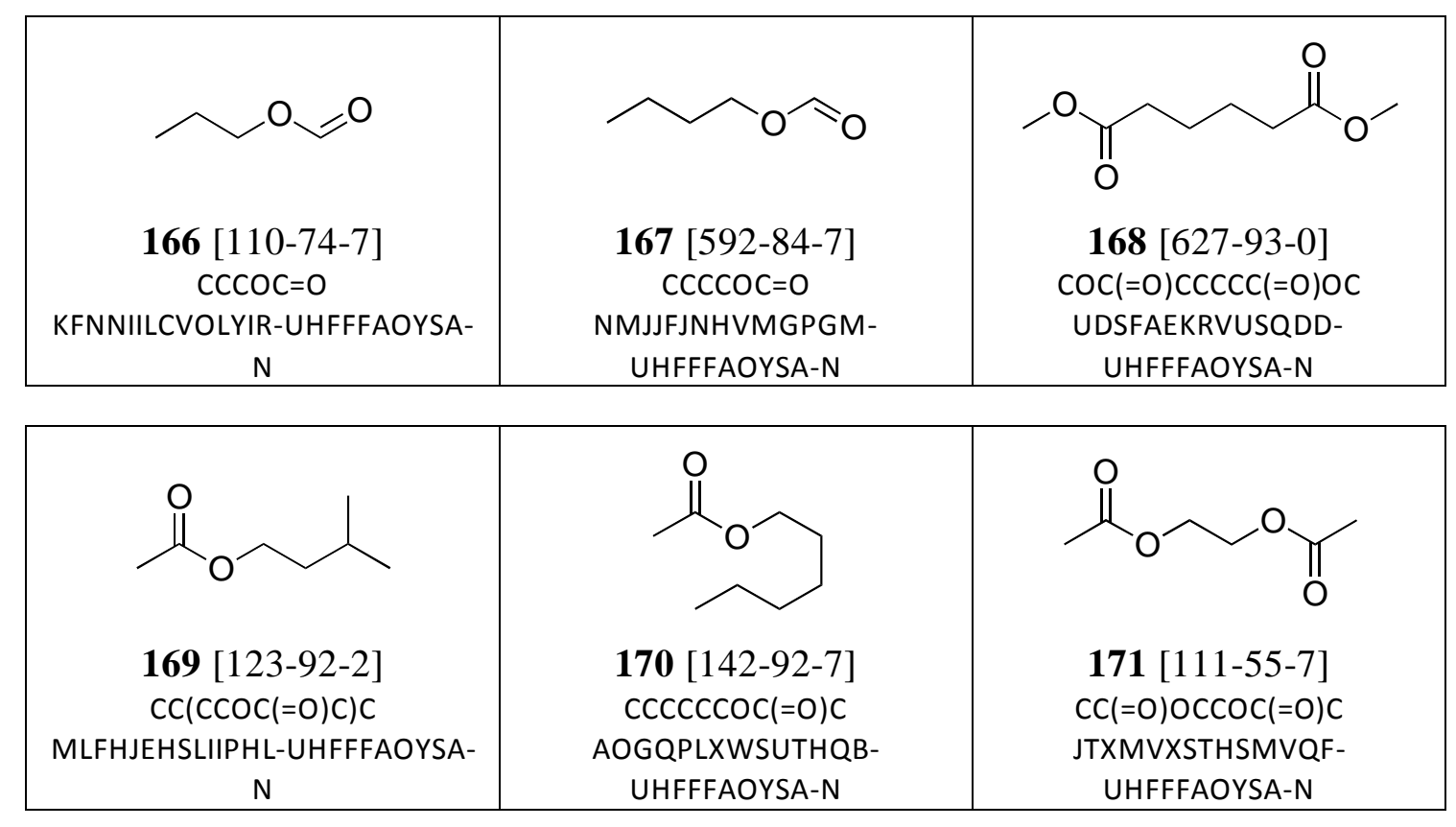

\begin{tabular}{|c|c|c|}
\hline $\begin{array}{c}\text { 172 [96-48-0] } \\
\text { O=C1CCCO1 } \\
\text { YEJRWHAVMIAJKC- } \\
\text { UHFFFAOYSA-N }\end{array}$ & $\begin{array}{c}173[149-73-5] \\
\text { COC(OC)OC } \\
\text { PYOKUURKVVELLB- } \\
\text { UHFFFAOYSA-N }\end{array}$ \\
\hline
\end{tabular}

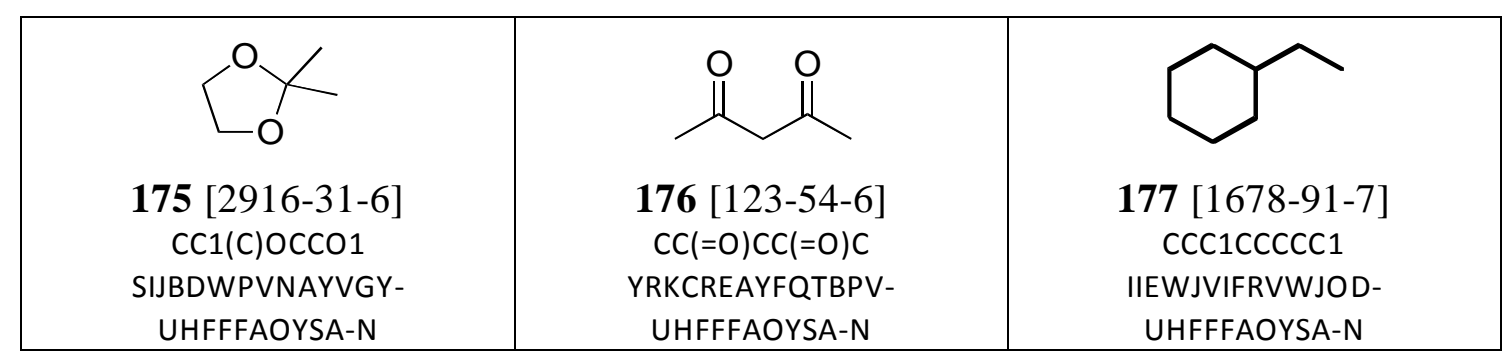

\begin{tabular}{|c|c|c|}
\hline $\begin{array}{l}\mathbf{1 7 8}[628-73-9] \\
\text { CCCCCC\#N } \\
\text { AILKHAQXUAOOFU- } \\
\text { UHFFFAOYSA-N }\end{array}$ & $\begin{array}{l}179[628-63-7] \\
\text { CCCCCOC }(=0) C \\
\text { PGMYKACGEOXYJE- } \\
\text { UHFFFAOYSA-N }\end{array}$ & $\begin{array}{c}180[554-12-1] \\
\text { CCC(=O)OC } \\
\text { RJUFJBKOKNCXHH- } \\
\text { UHFFFAOYSA-N }\end{array}$ \\
\hline
\end{tabular}




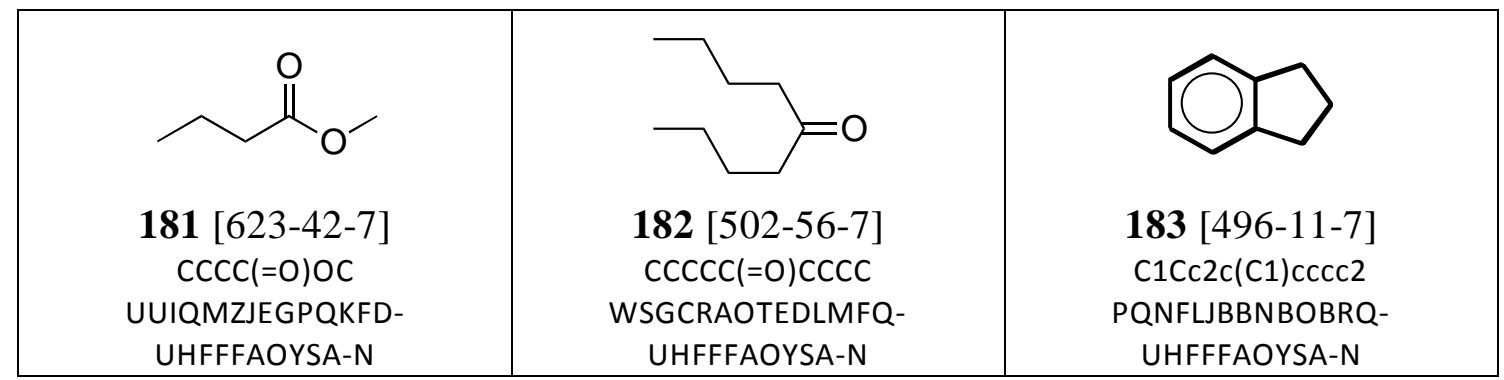

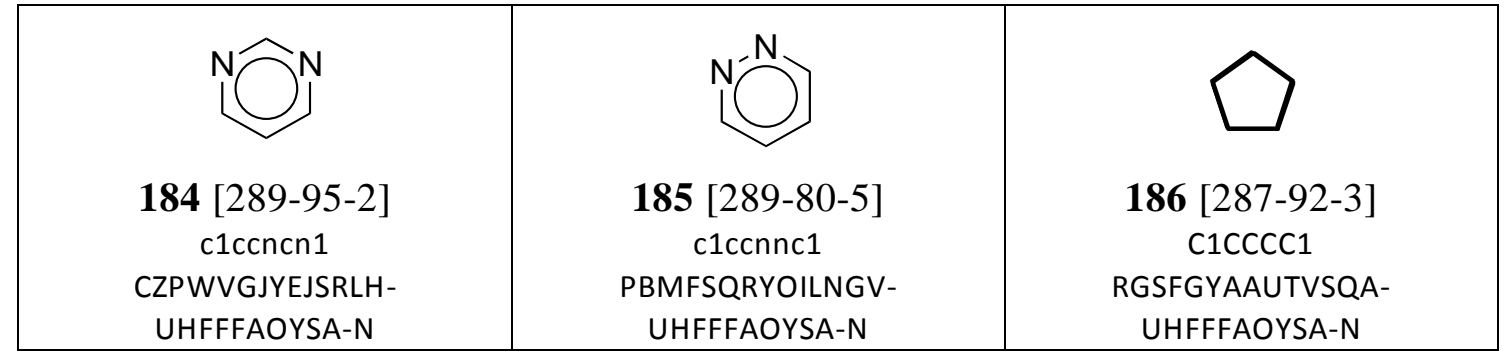

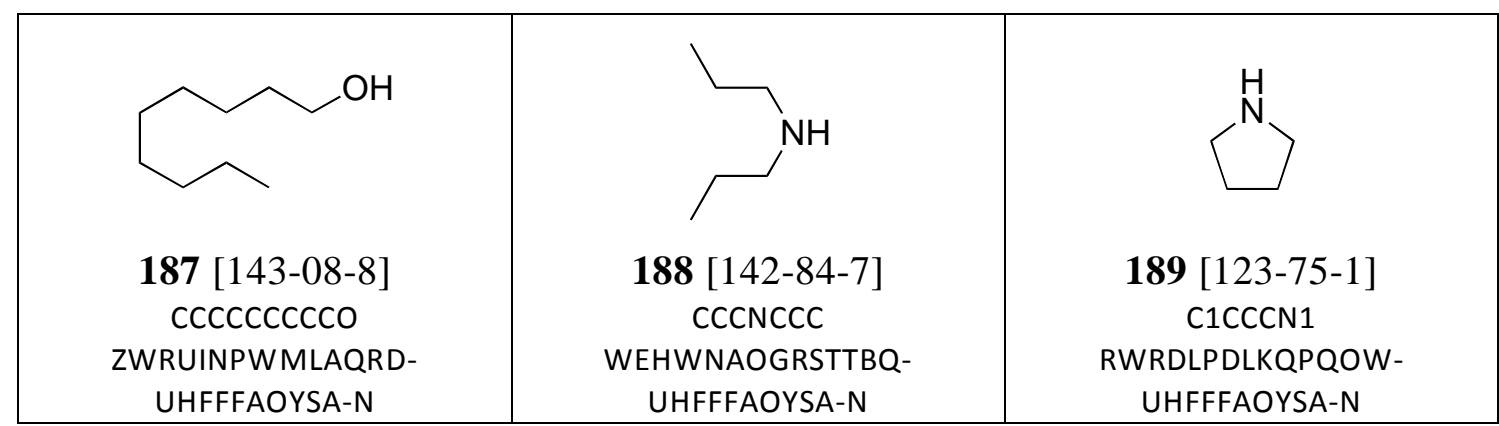

\begin{tabular}{|c|c|c|}
\hline $\begin{array}{c}190[123-42-2] \\
\text { CC }(=0) C C(O)(C) C \\
\text { SWXVUIWOUIDPGS- } \\
\text { UHFFFAOYSA-N }\end{array}$ & $\begin{array}{c}1 \\
191[120-92-3] \\
\text { O=C1CCCC1 } \\
\text { BGTOWKSIORTVQH- } \\
\text { UHFFFAOYSA-N }\end{array}$ & $\begin{array}{l}192[120-51-4] \\
\text { O=C(c1ccccc1)OCc1ccccc1 } \\
\text { SESFRYSPDFLNCH- } \\
\text { UHFFFAOYSA-N }\end{array}$ \\
\hline $\begin{array}{l}1 \\
193 \text { [111-92-2] } \\
\text { CCCCNCCCC } \\
\text { JQVDAXLFBXTEQA- } \\
\text { UHFFFAOYSA-N }\end{array}$ & $\begin{array}{c}194 \text { [111-70-6] } \\
\text { CCCCCCCO } \\
\text { BBMCTIGTTCKYKF- } \\
\text { UHFFFAOYSA-N }\end{array}$ & $\begin{array}{l}195 \text { [111-69-3] } \\
\text { N\#CCCCCCAN } \\
\text { BTGRAWJCKBQKAO- } \\
\text { UHFFFAOYSA-N }\end{array}$ \\
\hline
\end{tabular}




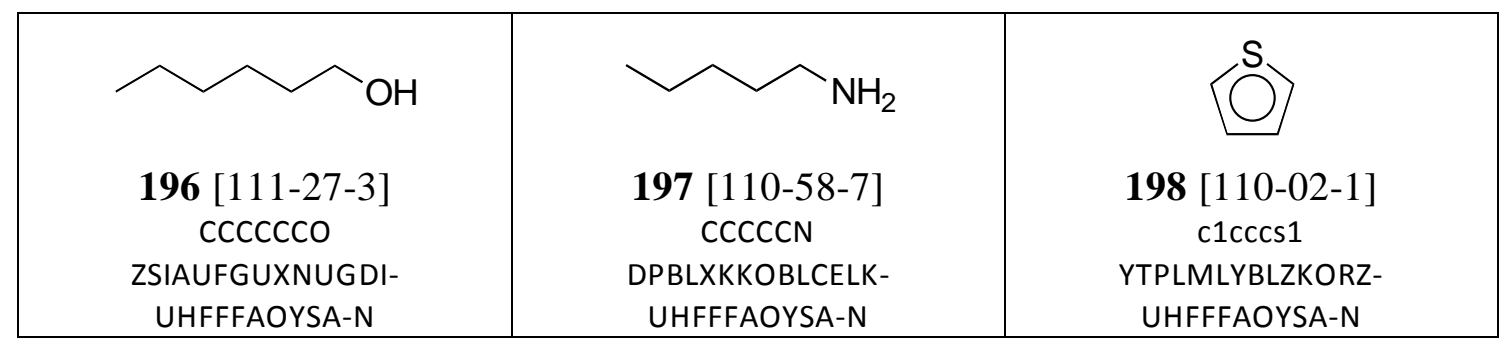

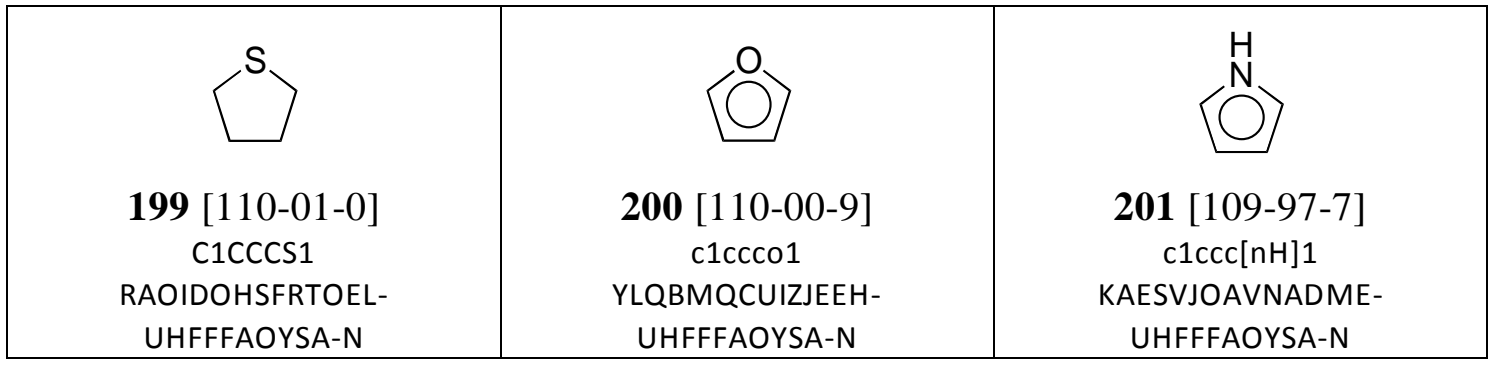

\begin{tabular}{|c|c|c|}
\hline $\begin{array}{c}\text { COCCN }[109-73-9] \\
\text { HQABUPZFAYXKJW- } \\
\text { UHFFFAOYSA-N }\end{array}$ & $\begin{array}{c}\mathbf{2 0 3}[109-60-4] \\
\text { CCCOC(=O)C } \\
\text { YKYONYBAUNKHLG- } \\
\text { UHFFFAOYSA-N }\end{array}$ & $\begin{array}{c}\mathbf{2 0 4}[108-91-8] \\
\text { NC1CCCCC1 } \\
\text { PAFZNILMFXTMIY- } \\
\text { UHFFFAOYSA-N }\end{array}$ \\
\hline
\end{tabular}

\begin{tabular}{|c|c|c|}
\hline & $\mathrm{O}^{-\mathrm{II}^{+}}$ & $\mathrm{O}$ \\
\hline 205 [108-18-9] & 206 [108-03-2] & $207[107-92-6]$ \\
\hline $\mathrm{CC}(\mathrm{NC}(\mathrm{C}) \mathrm{C}) \mathrm{C}$ & {$[\mathrm{O}-][\mathrm{N}+](=\mathrm{O}) \mathrm{CCC}$} & $\operatorname{cccc}(=0) 0$ \\
\hline $\begin{array}{l}\text { UAOMVDZJSHZZME- } \\
\text { UHFFFAOYSA-N }\end{array}$ & $\begin{array}{c}\text { JSZOAYXJRCEYSX-UHFFFAOYSA- } \\
\mathrm{N}\end{array}$ & $\begin{array}{l}\text { FERIUCNNQQJTOY- } \\
\text { UHFFFAOYSA-N }\end{array}$ \\
\hline
\end{tabular}

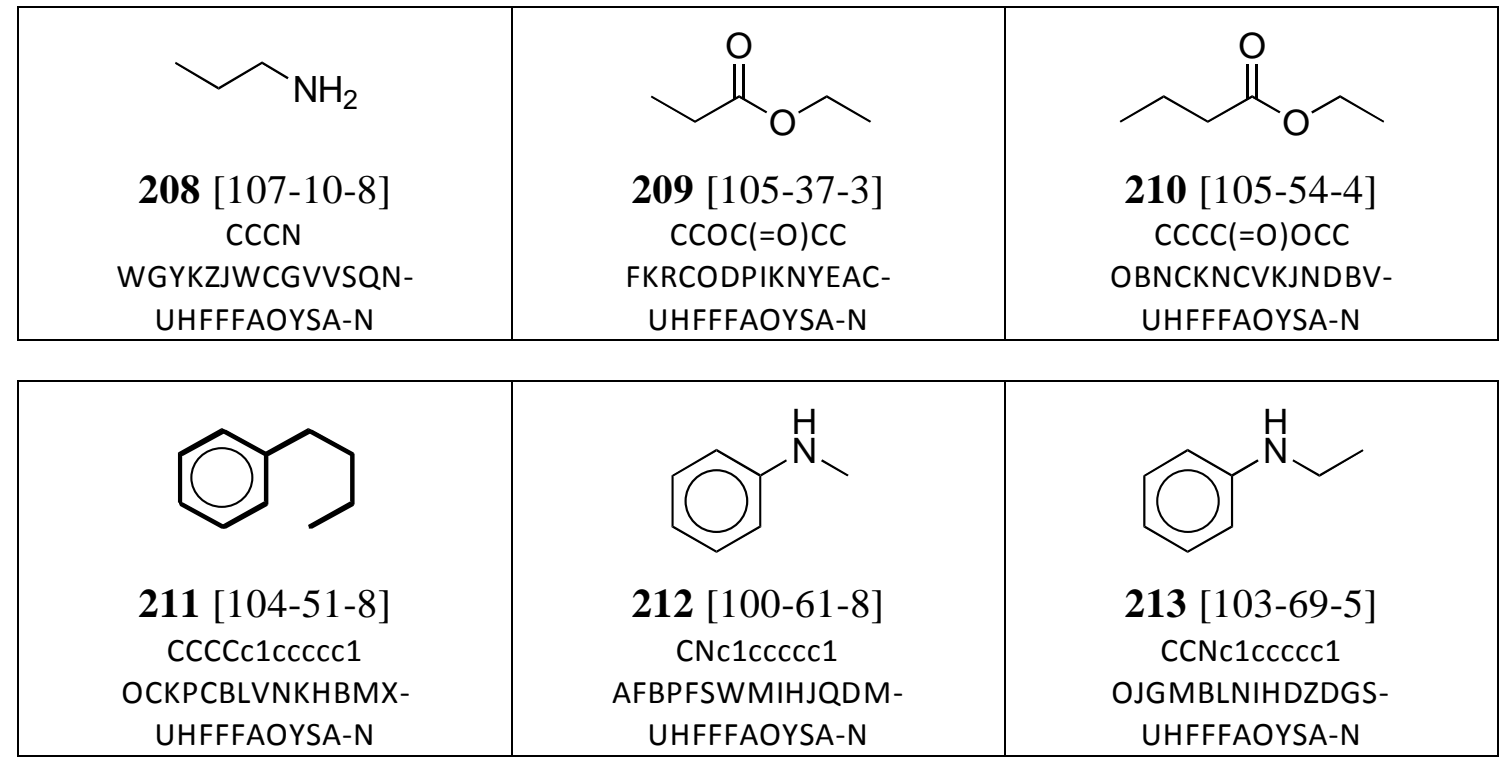




\begin{tabular}{|c|c|c|}
\hline $\begin{array}{c}\text { NCc1ccccc1 } \\
\text { WGQKYBSKWIADBV- } \\
\text { UHFFFAOYSA-N }\end{array}$ & $\begin{array}{c}\text { C15 [100-46-9] } \\
\text { CCc1ccccc1 } \\
\text { YNQLUTRBYVCPMQ- } \\
\text { UHFFFAOYSA-N }\end{array}$ & $\begin{array}{c}\mathbf{2 1 6}[98-82-8] \\
\text { CC(c1ccccc1)C } \\
\text { RWGFKTVRMDUZSP- } \\
\text { UHFFFAOYSA-N }\end{array}$ \\
\hline
\end{tabular}

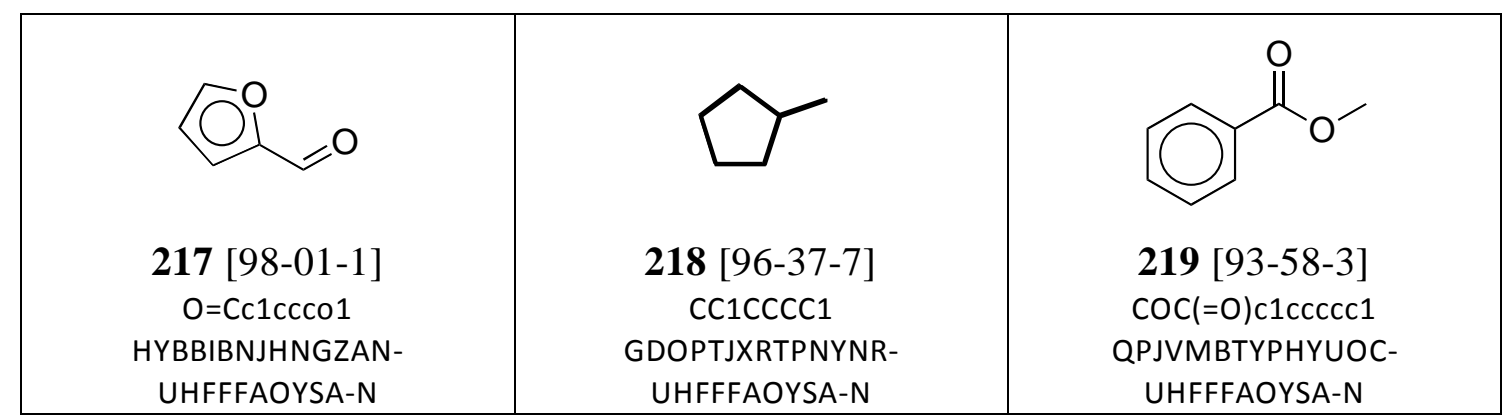

\begin{tabular}{|c|c|c|}
\hline &
\end{tabular}

\begin{tabular}{|c|c|c|}
\hline $\begin{array}{c}\mathrm{N23}_{\mathrm{N}^{-}}^{\mathrm{O}}[79-24-3] \\
\text { MO-][N+](=O)CC } \\
\text { MCSAJNNLRCFZED- } \\
\text { UHFFFAOYSA-N }\end{array}$ & $\begin{array}{l}224[79-00-5] \\
\begin{array}{c}\mathrm{ClCC}(\mathrm{Cl}) \mathrm{Cl} \\
\text { UBOXGVDOUJQMTN- } \\
\text { UHFFFAOYSA-N }\end{array} \\
\text { Cl }\end{array}$ & $\begin{array}{l}\mathbf{N ^ { 2 }} \mathrm{NH}_{2}[78-81-9] \\
\text { NCC(C)C } \\
\text { KDSNLYIMUZNERS- } \\
\text { UHFFFAOYSA-N }\end{array}$ \\
\hline $\begin{array}{l}\mathbf{2 2 6}[76-01-7] \\
\mathrm{CIC}(\mathrm{C}(\mathrm{Cl})(\mathrm{Cl}) \mathrm{Cl}) \mathrm{Cl} \\
\mathrm{BNIXVQGCZULYKV}- \\
\text { UHFFFAOYSA-N }\end{array}$ & $\begin{array}{c}\stackrel{\mathrm{NH}}{2}^{\mathrm{NH}_{2}} \\
\mathbf{2 2 7}[75-64-9] \\
\text { CC(N)(C)C } \\
\text { YBRBMKDOPFTVDT- } \\
\text { UHFFFAOYSA-N }\end{array}$ & $\begin{array}{c}228 \text { [75-31-0] } \\
\text { CC(N)C } \\
\text { JJWLVOIRVHMVIS- } \\
\text { UHFFFAOYSA-N }\end{array}$ \\
\hline
\end{tabular}




\begin{tabular}{|c|c|c|}
\hline $\begin{array}{l}229[57-55-6] \\
\text { OCC(O)C } \\
\text { DNIAPMSPPWPWGF- } \\
\text { UHFFFAOYSA-N }\end{array}$ & $\begin{array}{c}230 \text { [142-68-7] } \\
\text { C1CCCOC1 } \\
\text { DHXVGJBLRPWPCS- } \\
\text { UHFFFAOYSA-N }\end{array}$ & 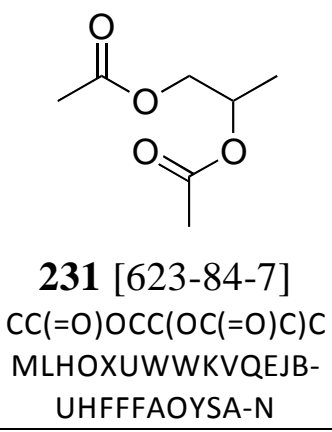 \\
\hline $\begin{array}{l}232[111109-77-4] \\
\text { COCC(OCC(OC)C)C } \\
\text { OQUIHNRSFOIOFU- } \\
\text { UHFFFAOYSA-N }\end{array}$ & $\begin{array}{l}\text { 233 [1087696-49-8] } \\
\text { O=C1CC[C@@H]2O[C@H]1OC2 } \\
\text { WHIRALQRTSITMI-UJURSFKZSA- } \\
\mathrm{N}\end{array}$ & $\begin{array}{c}234 \text { [22699-70-3] } \\
\text { FC(Oc1cccc1)(F)F } \\
\text { GQHWSLKNULCZGI- } \\
\text { UHFFFAOYSA-N }\end{array}$ \\
\hline
\end{tabular}

\begin{tabular}{|c|c|c|}
\hline $\begin{array}{c}235[109-02-4] \\
\text { CN1CCOCC1 } \\
\text { SJRJJKPEHAURKC-UHFFFAOYSA- } \\
\mathrm{N}\end{array}$ & $\begin{array}{c}236[34885-03-5] \\
\text { OCC1CCC (CC1)C } \\
\text { OSINZLLLLCUKJH-UHFFFAOYSA- } \\
\mathrm{N}\end{array}$ & $\begin{array}{c}237[107-46-0] \\
\text { C[Si](O[Si](C)(C)C)(C)C } \\
\text { UQEAIHBTYFGYIE- } \\
\text { UHFFFAOYSA-N }\end{array}$ \\
\hline
\end{tabular}

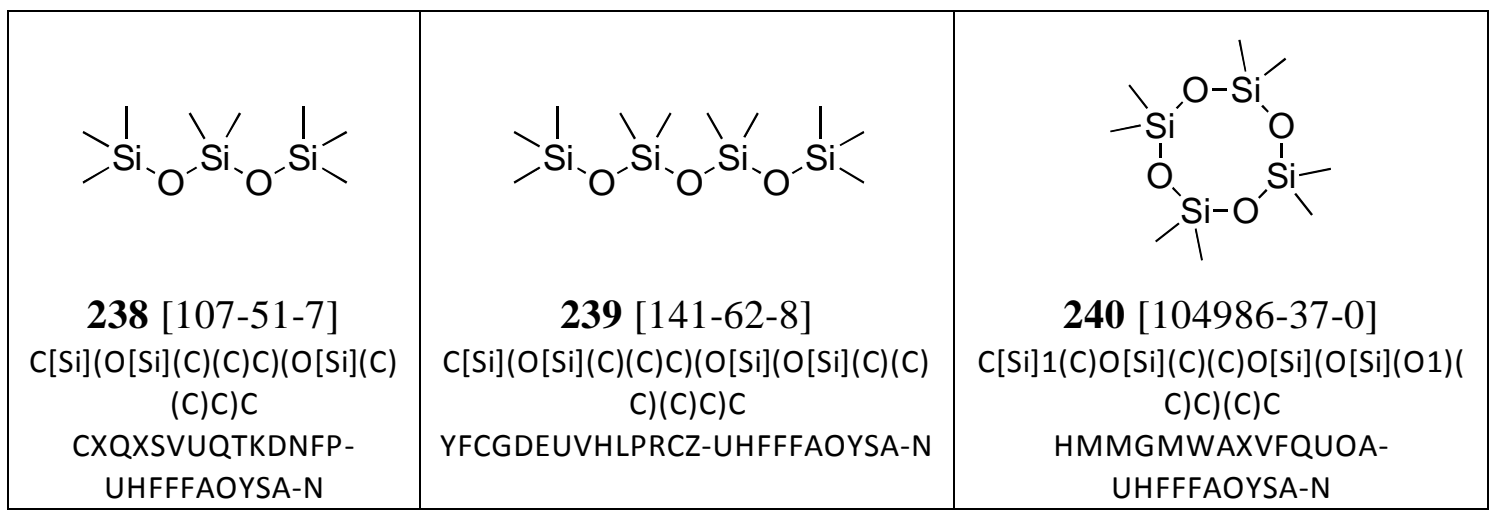

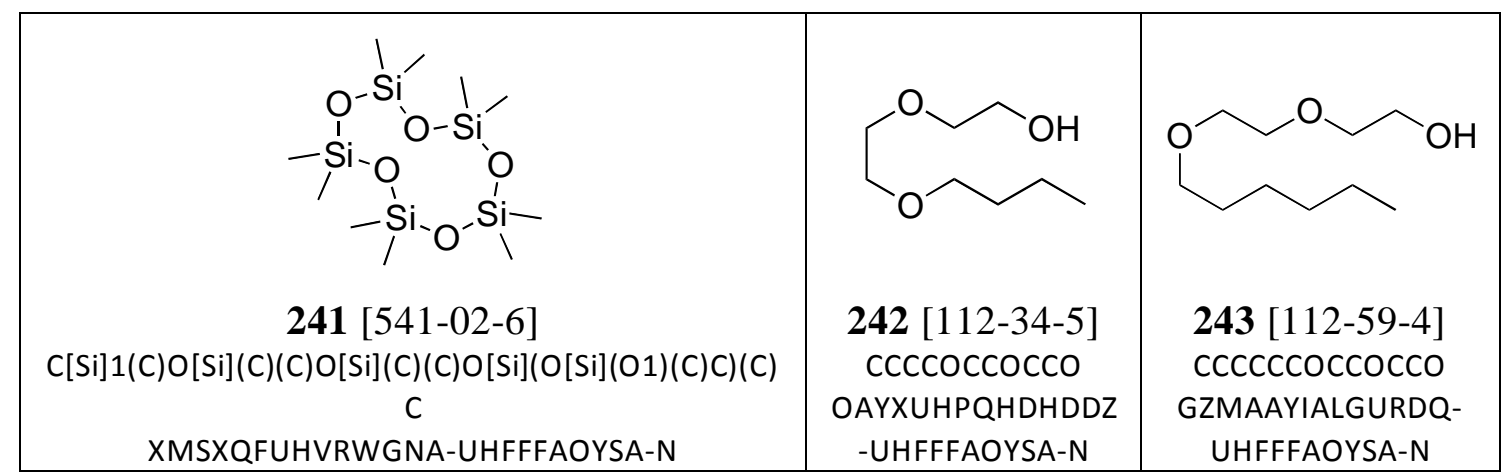




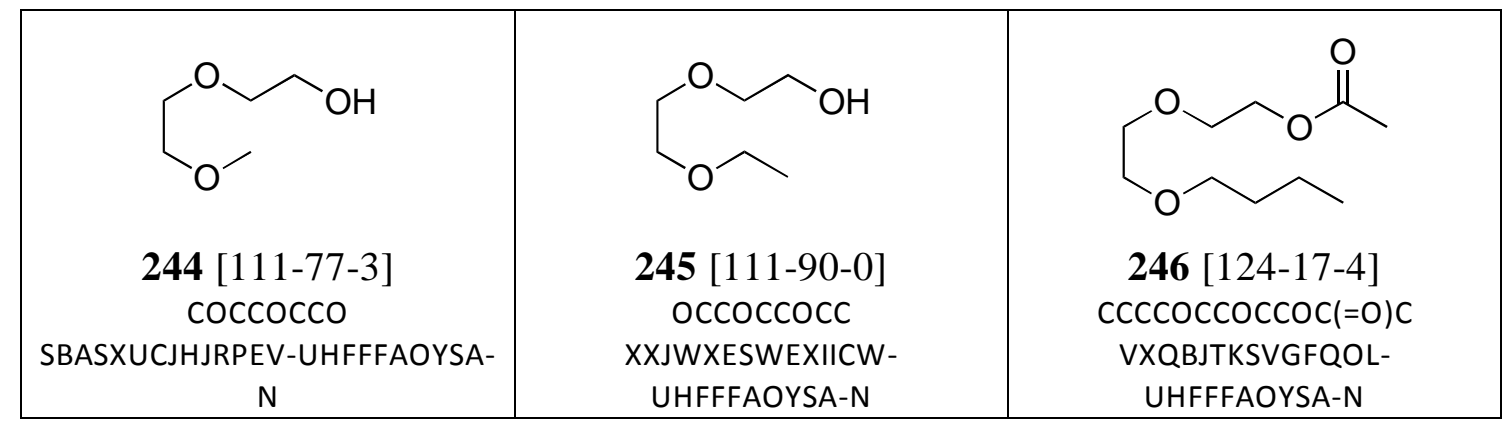

\begin{tabular}{|c|c|c|}
\hline $\begin{array}{c}\mathbf{2 4 7} \text { [2807-30-9] } \\
\text { CCCOCCO } \\
\text { YEYKMVJDLWJFOA- } \\
\text { UHFFFAOYSA-N }\end{array}$ & $\begin{array}{l}\mathbf{2 4 8}[112-07-2] \\
\text { CCCCOCCOC }(=0) C \\
\text { NQBXSWAWVZHKBZ- } \\
\text { UHFFFAOYSA-N }\end{array}$ & $\begin{array}{c}249 \text { [111-76-2] } \\
\text { CCCCOCCO } \\
\text { POAOYUHQDCAZBD- } \\
\text { UHFFFAOYSA-N }\end{array}$ \\
\hline
\end{tabular}

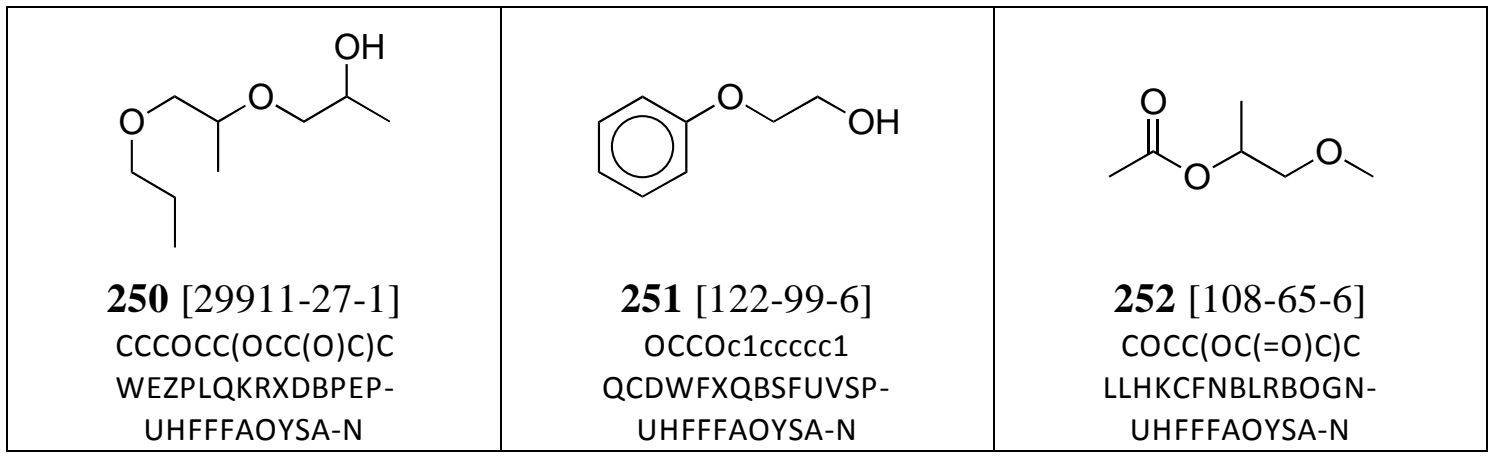

\begin{tabular}{|c|c|c|}
\hline $\begin{array}{l}\mathbf{2 5 3}[623-84-7] \\
\begin{array}{l}\text { CC(=O)OCC(OC(=O)C)C } \\
\text { MLHOXUWWKVQEJB- } \\
\text { UHFFFAOYSA-N }\end{array}\end{array}$ & $\begin{array}{l}254 \text { [107-98-2] } \\
\text { COCC(O)C } \\
\text { ARXJGSRGQADJSQ- } \\
\text { UHFFFAOYSA-N }\end{array}$ & $\begin{array}{l}255 \text { [5131-66-8] } \\
\text { CCCCOCC(O)C } \\
\text { RWNUSVWFHDHRCJ- } \\
\text { UHFFFAOYSA-N }\end{array}$ \\
\hline $\begin{array}{l}256[1569-01-3] \\
\text { CCCOCC(O)C } \\
\text { FENFUOGYJVOCRY- } \\
\text { UHFFFAOYSA-N }\end{array}$ & $\begin{array}{c}\mathbf{2 5 7}[770-35-4] \\
\text { CC(COc1ccccc1)O } \\
\text { IBLKWZIFZMJLFL-UHFFFAOYSA- } \\
\mathrm{N}\end{array}$ & $\begin{array}{c}\text { l } \\
\mathbf{2 5 8}[25498-49-1] \\
\text { COCC(OCC(OCC(O)C)C)C } \\
\text { HPFDGTFXAVIVTH- } \\
\text { UHFFFAOYSA-N }\end{array}$ \\
\hline
\end{tabular}




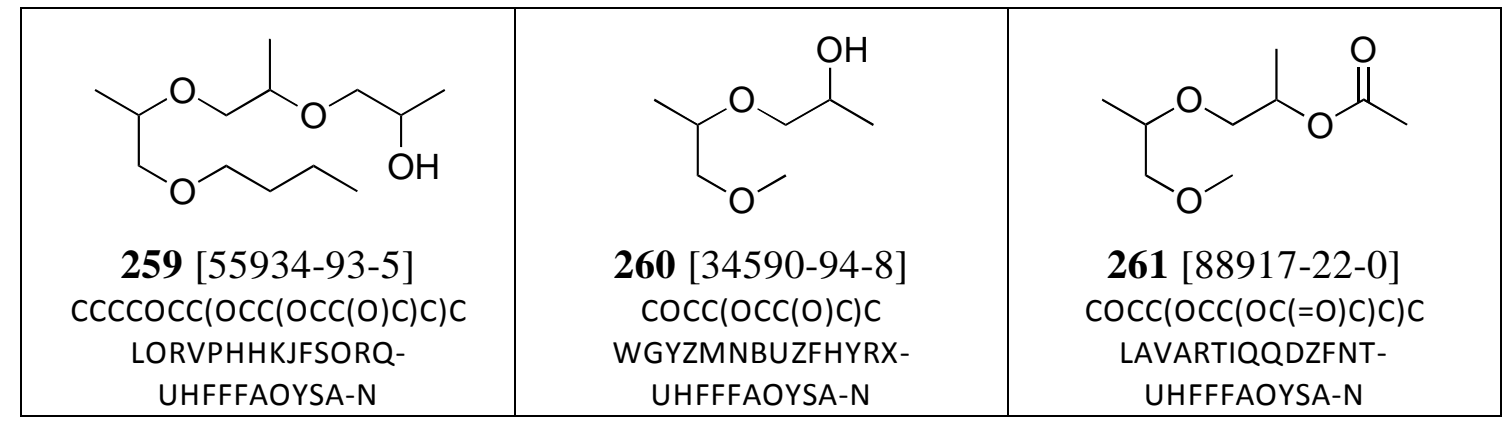

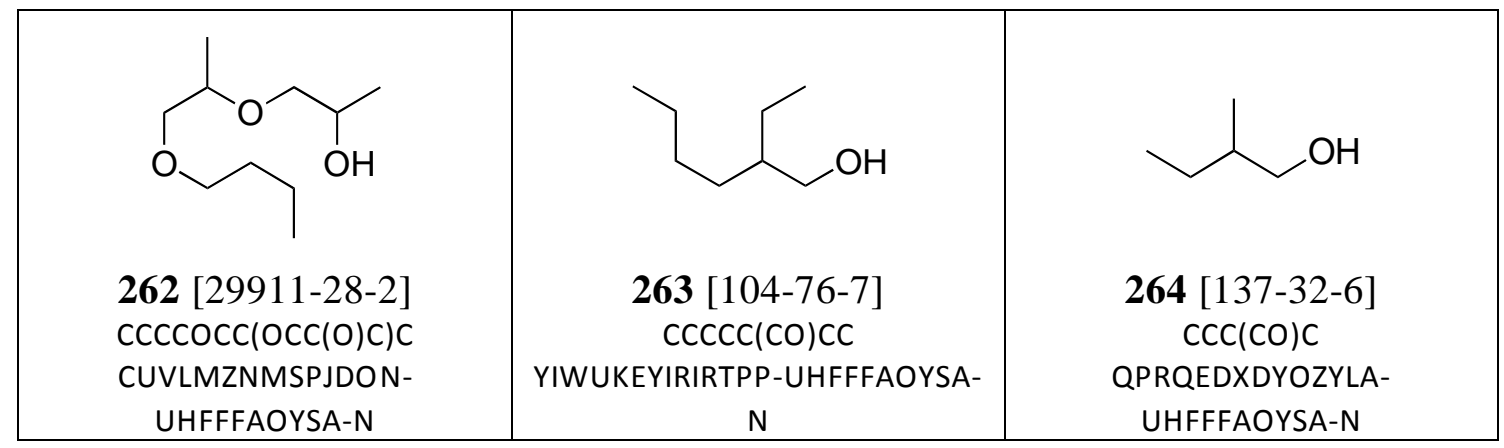

\begin{tabular}{|c|c|c|}
\hline & & \\
\hline 265 [108-11-2] & 266 [106-36-5] & 267 [763-69-9] \\
\hline $\mathrm{CC}(\mathrm{CC}(\mathrm{O}) \mathrm{C}) \mathrm{C}$ & $\operatorname{cccoc}(=0) \operatorname{cc}$ & $\operatorname{ccoccc}(=0) O C c$ \\
\hline $\begin{array}{l}\text { WVYWICLMDOOCFB- } \\
\text { UHFFFAOYSA-N }\end{array}$ & $\begin{array}{c}\text { MCSINKKTEDDPNK- } \\
\text { UHFFFAOYSA-N }\end{array}$ & $\begin{array}{l}\text { BHXIWUJLHYHGSJ- } \\
\text { UHFFFAOYSA-N }\end{array}$ \\
\hline
\end{tabular}

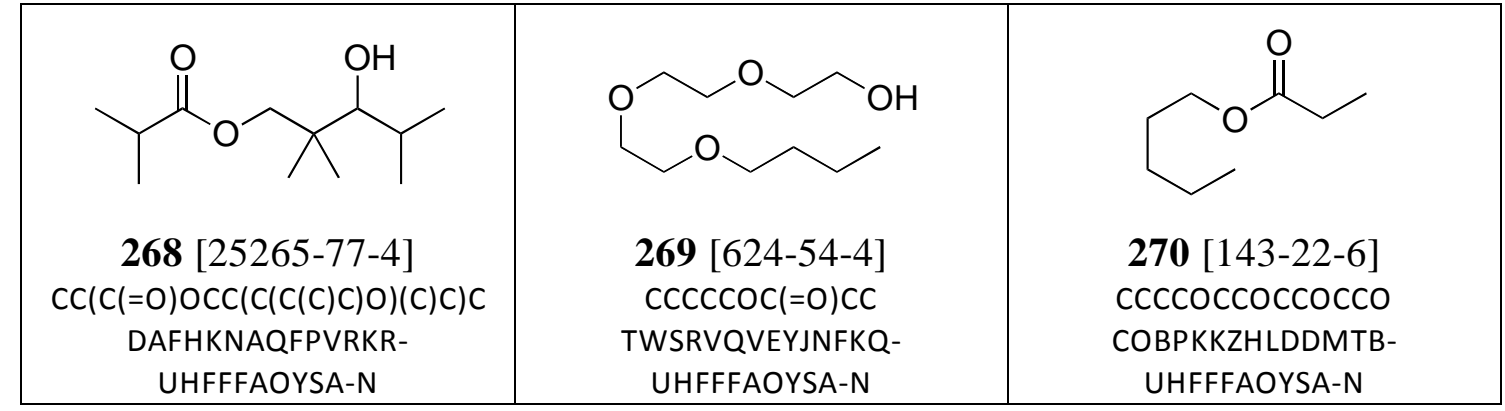

\begin{tabular}{|c|c|}
$271[111109-77-4]$ \\
$\begin{array}{c}\text { COCC(OCC(OC)C)C } \\
\text { OQUIHNRSFOIOFU- } \\
\text { UHFFFAOYSA-N }\end{array}$ \\
$\begin{array}{c}\text { CC(=O)OC(COC(=O)C)COC }(=0) C \\
\text { URAYPUMNDPQOKB- } \\
\text { UHFFFAOYSA-N }\end{array}$ \\
\hline
\end{tabular}




\section{Physical Properties}

Physical properties from a number of data sources, including commercially available database programs ${ }^{1,2}$ web resources, ${ }^{3}$ commercial suppliers data sheets and primary literature sources have been compiled for the principal component analysis, and property based filtering and information within the Solvent Selection Tool.

The melting points, boiling points and densities have been compiled primarily for filtering of the solvents according to the desired reaction/process conditions, although in principal they values reflect the strength of the solvent - solvent interactionsx.

The molar volume is a molar size property and is commonly used to correct for the effect of molecular size upon dispersion. The refractive index provides information regarding the polarisibility of the solvent to an electric field. Whilst the refractive index can be measured with any light source, we have opted for the most commonly used wavelength of the sodium emission D-line $(598 \mathrm{~nm})$ at $20^{\circ} \mathrm{C}$. The refractive index is linked to the molecular polarisability through the Lorentz-Lorenz equation:

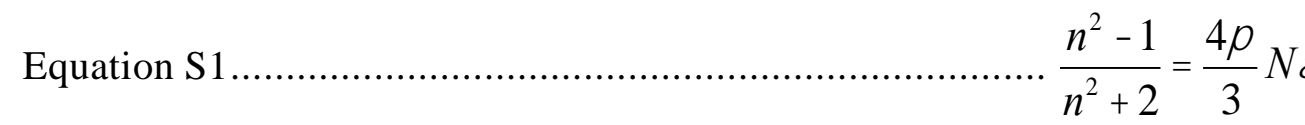

Where $n$ is the refractive index, $N$ is the number of molecules per unit volume and $\alpha$ is the polarisibility.

The dielectric constant and dipole moment are physical properties that are commonly associated with the polarity (charge separation) of the solvent.

The Hildebrand solubility parameter $(\delta)^{4}$ is the amount of work required to separate the solvent molecules (i.e. disruption and reorganisation of solvent - solvent interactions) to create a cavity of suitable size to accommodate the solute (the solvent cohesive energy). It is defined as the square root of the change of internal energy of vaporisation divided by the molar volume. Whilst the Hildebrand parameter can be calculated from the data we have compiled, we have opted to quote data from database/literature sources.

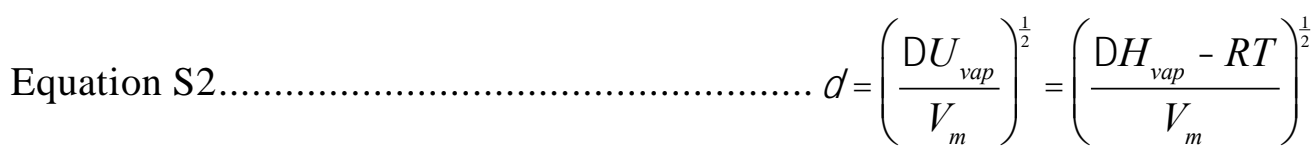

The Hildebrand solubility parameter does not differentiate between the different types of solvent - solvent interaction. The Hansen parameters ${ }^{5}$ attempts to separate the solvent cohesive energy into its component parts of dispersion $\left(\delta_{d}\right)$, dipolar $\left(\delta_{p}\right)$ and hydrogen bonding $\left(\delta_{h}\right)$. The conventional units for the Hildebrand and Hansen

SMSWin, "Solvents, Melts and Solutions", Morrison J. W.; Version 2.0.

“Aspen Properties”, Aspen Technology, Inc., Version 8.4.

ChemSpider [Online] www.ChemSpider.com

$4 \quad$ Hildebrand J. H.; Prausnitz J. M.; Scott R. L., Regular and Related Solutions, Van NostrandReinhold, Princeton, 1970. and Their Importance in Surface Coating Formulation. Copenhagen: Danish Technical Press, 1967. 
solubility parameters are $\left(\mathrm{cal} \mathrm{cm}^{-3}\right)^{0.5}$ which have been used herein. More recent determinations use units of either $\left(\mathrm{J} \mathrm{cm}^{-3}\right)^{0.5}$ or $\mathrm{MPa}^{0.5}$. To convert from these units, the numeric values are divided by the square root of 4.184 .

Four solvatochromic parameters have been used, Richardt's $E_{T}^{N}$ and Kamlet-Taft $\pi^{*}$, $\alpha$ and $\beta$ values.

Solvatochromism ${ }^{6}$ is used to describe the effect of pronounced shifts in the position and intensity of a UV-Vis absorption band following a change of the solvent media. When absorption spectra are recorded in a range of solvents of different polarities and hydrogen-bonding donor and acceptor strengths, a various polarity and hydrogenbonding empirical scales can be derived.

A number of solvatochromic dyes systems have been evaluated; however, two sets have the greatest portion of evaluated solvents. The Dimroth - Reichardt dye is probably the most extensive studied with over 350 measurements in pure solvents, and several thousand measurements in binary mixtures, electrolyte solutions and micro-heterogeneous systems (surfactants, micelles, vesicles, etc.). The dye undergoes exceptional large hypsochromic shifts providing a measure of the solvent's polarity (dipolarity-polarisability). The $E_{T}^{N}$ scale is normalised between 0 (tetramethylsilane) and 1 (water).

There are a number of limitations of the Dimroth - Reichardt dye. The first is that the solvatochromism effect is disrupted in acidic media due to the protonation of the phenolate oxygen. Whilst being relatively soluble in most organic solvents the dye is poorly soluble in water and virtually insoluble perfluoronated solvents, thereby limiting its use for some classes of solvent. Finally, the dye measures dipolaritypolarisability and doesn't differentiate Lewis acid/basicity.

To address this latter issue, multi-parameter correlations using a series of dyes have been developed to separate different aspects of the solvent-solute interactions: dipolarity, polarisability, Lewis acidity and Lewis basicity. Kamlet and Taft developed the first multi-parameter scales, based upon the following equation:

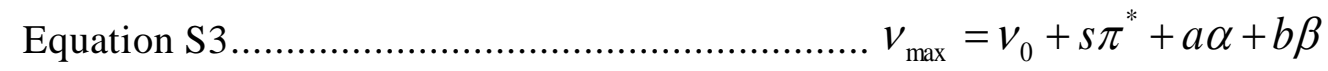

The $\pi^{*}$ scale is presumed to represent the dipolarity-polarisability interactions in the absence of other strong forces such as hydrogen-bonding or dipole interactions. It is normalised between 0 (cyclohexane) and 1 (DMSO). The $\alpha$ scale is a measure of the solvent's hydrogen-bonding donor (HBD) acidity and is normalised from 0 (cyclohexane) to 1 (methanol). Similarily the $\beta$ scale is for the solvent's hydrogenbonding acceptor (HBA) basicity, and is normalised from 0 (cyclohexane) to 1 (hexamethylphosporamide).

The Gutmann Donor Number is a quantitative measure of Lewis Basicity. ${ }^{7}$ The donor number is defined as the negatively enthalpy $\left(\mathrm{kcal} \mathrm{mol}^{-1}\right)$ of reaction, for the

\footnotetext{
$6 \quad$ a) Nigam, S.; Rutan, S.; Applied Spectroscopy, 2001, 55, 362A-370A. b) Reichardt, C.; Chem. Rev., 1994, 94, 2319-2358.

7 Gutmann V.; Coord. Chem. Rev., 1976, 18, 225-255.
} 
formation of a 1:1 complex of the solvent and antimony pentachloride in a dilute 1,2dichloroethane solution. ${ }^{8}$,

\section{Scheme S1}

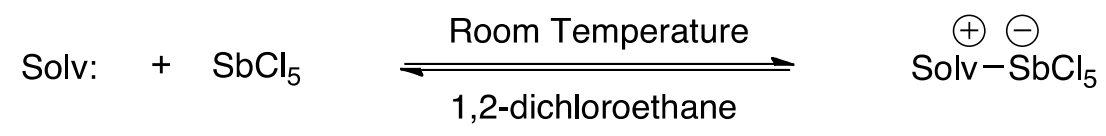

The octanol - water partition $\left(\log P_{o w}\right)$ coefficient proves a measure of lipophilicity (hydrophobicity).

Michael Abraham and co-workers have developed a general solvation equation that correlates the solution properties of solute $(S P)$ with five parameters according to the following equation. ${ }^{10}$

$$
\text { Equation S4. }
$$$$
\log S P=c+e E+s S+a A+b B+v V
$$

In this equation, $E$ is the excess molar refraction which is obtained from the refractive index of the compound. $S$ is the dipolarity/polarisability which can be obtained either from GC measurements using a polar stationary phase or more commonly from the partition coefficients of solvent/water systems. The parameters $A$ and $B$ are the overall hydrogen bond acidity and basicity, which can again be obtained from partition coefficient measurements. $V$ is the McGowan volume which can be readily calculated from bond and atom contributions. These parameters represent the different aspects of the solute influence on various solute/solvent interactions.

The coefficients of the equation, $c, e, s, a, b$ and $v$, are obtained using multiple linear regression against known $S P$ values. These coefficients correspond to the complimentary effect of the solvent system to the solute and can be regarded as system constants which characterise the nature of the solvent - solute interactions with respect to the system under consideration. The $e$-coefficient represents the tendency of the phase to interact with the solute through $\pi$ - and $n$-electrons pairs. The $s$-coefficient represents the tendency of the phase to interact with dipolar/polarizable solutes. The $a$-coefficient denotes the hydrogen bond basicity of the phase on the basis that basic solutes will interact with acidic solvents (Lewis and Brønsted Acids and Bases). Similarly the $b$-coefficient is a measure of the hydrogen bond acidity of the phase. The $v$-coefficient is a measure of the hydrophobicity of the phase and it describes the dispersion interactions and cavitation forces.

This approach has been applied to a diverse range of applications including solubility, ${ }^{11}$ liquid-liquid extraction ${ }^{12}$ as well as biological processes like human intestinal absorption ${ }^{13}$ and blood-brain distribution. ${ }^{14}$

8 In principle, the donor number can be directly calculated using quantum mechanical methods. However, to reduce the computational burden, boron trifluoride could be used as the Lewis acid. Gal and Maria have developed the experimental protocol [reference 9]. This method appears to be more reliable the Guttman method. At this time, we have chosen to use the Gutmann experimental values of the donor number. 
Several authors have attempted to calculate values of $S, A$ and $B$ using quantum mechanical methods ${ }^{15}$ so that in silico screening approaches can be used to estimate desirable properties.

The auto-ignition temperatures have been included for plant accommodation purposes. The UK Electric Equipment Temperature Classification (surface temperature) has been used to define the T-Rating according to the following criteria. $^{16}$

$$
\text { Temperature Class }=\left\{\begin{array}{l}
\text { Temperature } \leq 450^{\circ} \mathrm{C} \rightarrow T 1 \\
300^{\circ} \mathrm{C} \leq \text { Temperature }<450^{\circ} \mathrm{C} \rightarrow T 2 \\
200^{\circ} \mathrm{C} \leq \text { Temperature }<300^{\circ} \mathrm{C} \rightarrow T 3 \\
135^{\circ} \mathrm{C} \leq \text { Temperature }<200^{\circ} \mathrm{C} \rightarrow T 4 \\
100^{\circ} \mathrm{C} \leq \text { Temperature }<135^{\circ} \mathrm{C} \rightarrow T 5 \\
85^{\circ} \mathrm{C} \leq \text { Temperature }<100^{\circ} \mathrm{C} \rightarrow T 6
\end{array}\right.
$$

A Red/Amber/Green visualise classification has been used (Red for T5 and T6, Amber for T3 and T4, and Green for T1 and T2).

An assessment of each solvents' flammability, based upon the closed cup, flash points and the US National Fire Protection Agency flammability classification has been used. ${ }^{17}$

12 a) Abraham, M. H.; Chadha, H. S.; Whinting, G. S.; Mitchell, R. C. J. Pharm. Sci. 1994, 83, 1085-1100. b) Abraham, M. H.; Zissimos, A. M.; Acree, W. E. Phys. Chem. Chem. Phys. 2001, 3, 3732-3736. D.; Beck, G.; Sherbone, B.; Cooper, I.; Platts, J. A., J. Pharm. Sci. 2001, 90, 749-784.

Platts, J. A.; Abraham, M. H.; Zhao, Y. H.; Hersey, A.; Ijaz, L.; Butina, D. Eur. J. Med. Chem. 2001, 36, 719-730.

a) Svozil, D.; Sevecik, J. G. K.; Kvasnicka, V. J. Chem. Inf. Comput. Sci., 1997, 37, 338-342. b) Lamarche, O.; Platts, J. A.; Hersey, A., Phys. Chem. Chem. Phys., 2001, 3, 2747-2753. c) Platts, J. A., Phys. Chem. Chem. Phys., 2000, 2, 973-980. d) Platts, J. A. Phys. Chem. Chem. Phys., 2000, 2, 3115-3120. e) Lamarche, O.; Platts, J. A. Phys. Chem. Chem. Phys. 2003, 5, 677-684. f) Platts, J. A.; Butina, D.; Ahraham, M. H.; Hersey, J. Chem. Inf. Comput. Sci., 1999, 39, 835-845. g) Klamt, A. J. Phys. Chem. 1995, 99, 2224-2235. h) Zissimos, A. M.; Abraham, M. H.; Klamt, A.; Eckert, F.; Wood, J. J. Chem. Inf. Comput. Sci. 2002, 42, 1320 1331. i) Katritzky, A. R.; Maran, U.; Lobanov, V. S.; Karelson, M. J. Chem. Inf. Comput. Sci., 2000, 40, 1-18. j) Jesués Jover, Ramoén Bosque, and Joaquim Sales J. Chem. Inf. Comput. Sci. 2004, 44, 1098-1106

16 [Online]. http://www.hse.gov.uk/comah/sragtech/techmeasareaclas.htm

$17 \quad$ National Fire Protection Association's publication NFPA 30, Flammable and Combustible Liquids Code. 29 CFR 1910.106. [Online]. Available. https://www.osha.gov/dte/library/flammable_liquids/flammable_liquids.html Accessed $19 / 07 / 2014$ 
Flammability Class $=\left\{\begin{array}{l}\text { Flash Point } \geq 93.3^{\circ} \mathrm{C} \rightarrow \text { Class IIIB } \\ 60^{\circ} \mathrm{C} \leq \text { Flash Point }<93.3^{\circ} \mathrm{C} \rightarrow \text { Class IIIA } \\ 37.8^{\circ} \mathrm{C} \leq \text { Flash Point }<60^{\circ} \mathrm{C} \rightarrow \text { Class II } \\ 22.8^{\circ} \mathrm{C} \leq \text { Flash Point }<37.8^{\circ} \mathrm{C} \rightarrow \text { Class IC } \\ 22.8^{\circ} \mathrm{C} \leq \text { Flash Point }<37.8^{\circ} \mathrm{C} \text { and Boiling Point } \geq 37.8 \rightarrow \text { Class IB } \\ 22.8^{\circ} \mathrm{C} \leq \text { Flash Point }<37.8^{\circ} \mathrm{C} \text { and Boiling Point }<37.8 \rightarrow \text { Class IA }\end{array}\right.$

A Red/Amber/Green visualise classification has been used (Red for Classes IA, IB and IC, Amber for Class II, and Green for Classes IIIA and IIIB).

The vapour pressure $(\mathrm{mmHg})$, at $20^{\circ} \mathrm{C}$, have been calculated from an extended form of the Antoine equation. ${ }^{18}$

Equation S5 $P=\exp A+\frac{B}{T+C} \div+D T+E \ln (T)$

Where $P$ is the vapour pressure $\left(N m^{2}\right), T$ is the temperature $(\mathrm{K})$ and $A, B, C, D$ and $E$ are the Antoine constants. The pressure was converted in units of $\mathrm{mmHg}$ by multiplying by 0.00750061683 to be compatible with the environmental VOC potential scoring system.

$$
\text { VOC Potential }=\left\{\begin{array}{l}
\text { Vapour Pressure }<20 \mathrm{mmHg} \rightarrow \text { Group } 1 \\
20 \mathrm{mmHg} \leq \text { Vap our Pressure }<150 \mathrm{mmHg} \\
\text { Vapour Pressure } \geq 150 \mathrm{mmHg} \rightarrow \text { Group } 3
\end{array} \rightarrow \text { Group } 2\right.
$$

The solvent viscosity at $20^{\circ} \mathrm{C}$ has been included to provide information regarding the difficulty or ease of filtration. The following classification has been used:

$$
\text { Viscosity Class }=\left\{\begin{array}{l}
\text { Viscosity }<1 \mathrm{cP} \rightarrow \text { Class } 0 \\
1 \mathrm{cP} \leq \text { Viscosity }<10 \mathrm{cP} \rightarrow \text { Class } 1 \\
10 \mathrm{cP} \leq \text { Viscosity }<100 \mathrm{cP} \rightarrow \text { Class } 2 \\
\text { Viscosity }<100 \mathrm{cP} \rightarrow \text { Class } 3
\end{array}\right.
$$

18 Antoine, C. Comptes Rendus des Séances de l'Académie des Sciences, 1888, 107, 681-684, 778-780 and 836-837. 


\section{Table S1 Summary of Physical Property Sources and Units.}

Primary Data Sources

\begin{tabular}{|c|c|c|c|c|c|c|c|}
\hline Property & Units & 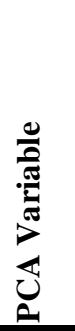 & $\sum_{i=0}^{n}$ & 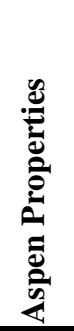 & 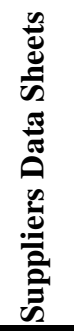 & 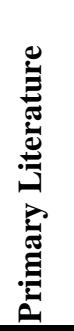 & 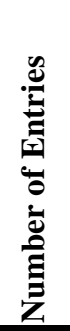 \\
\hline Melting Point & ${ }^{\circ} \mathrm{C}$ & • & • & & $\cdot^{\mathrm{a}}$ & & 253 \\
\hline Boiling Point & ${ }^{\circ} \mathrm{C}$ & - & - & & - a & & 271 \\
\hline Density ${ }^{\mathrm{b}}$ & $\mathrm{g} \mathrm{cm}^{-3}$ & - & - & & $\cdot{ }^{\mathrm{a}}$ & . ${ }^{c}$ & 272 \\
\hline Refractive Index ${ }^{d}$ & N/A & - & • & & - a & $\bullet^{\mathrm{c}}$ & 253 \\
\hline Dipole Moment & Debye & - & - & & &.$^{c}$ & 224 \\
\hline Dielectric Constant & N/A & - & - & & & . $^{\mathrm{c}}$ & 160 \\
\hline Hildebrand Solubility Parameter & $\left(\mathrm{cal} \mathrm{cm}^{-3}\right)^{0.5}$ & - & - & & &.$^{c}$ & 221 \\
\hline Hansen Parameter (Polar) & $\left.(\mathrm{cal} \mathrm{cm})^{-3}\right)^{0.5}$ & . & . & & . & & 130 \\
\hline Hansen Parameters (Dispersion) & $\left(\mathrm{cal} \mathrm{cm}^{-3}\right)^{0.5}$ & • & - & & - & & 130 \\
\hline Hansen Parameters (Hydrogen-Bonding) & $\left(\mathrm{cal} \mathrm{cm}^{-3}\right)^{0.5}$ & - & - & & • & & 130 \\
\hline Solvatochromic $-\mathrm{E}_{\mathrm{T}} \mathrm{N}^{2}$ & N/A & - & - & & & $\bullet^{\mathrm{c}}$ & 152 \\
\hline Solvatochromic $-\pi^{*}$ & N/A & • & • & & & $e^{\mathrm{e}}$ & 90 \\
\hline Solvatochromic $-\alpha$ & N/A & • & - & & & $e^{\mathrm{e}}$ & 82 \\
\hline Solvatochromic $-\beta$ & N/A & - & - & & &.$^{\mathrm{e}}$ & 79 \\
\hline Gutmann Donor Number & $\mathrm{kcal} \mathrm{mol}^{-1}$ & • & - & & & - c,f & 109 \\
\hline $\log$ Pow & N/A & - & - & & • a & & 195 \\
\hline Molar Volume & $\mathrm{cm}^{3} \mathrm{~mol}^{-1}$ & - & & & & • $^{\mathrm{c}}$ & 230 \\
\hline Abraham Parameters - AH & N/A & - & & & &. $\mathrm{g}$ & 125 \\
\hline Abraham Parameters - BH & N/A & - & & & & •g & 125 \\
\hline Abraham Parameters - R2 & N/A & - & & & & . g & 125 \\
\hline Abraham Parameters - Pi & N/A & - & & & &. $\mathrm{g}$ & 125 \\
\hline Abraham Parameters $-\mathrm{L}^{16 \mathrm{~h}}$ & N/A & • & & & &.$^{\mathrm{i}}$ & 102 \\
\hline Abraham Parameters $-V_{x}$ & N/A & • & & & & $\bullet^{\mathrm{j}}$ & 272 \\
\hline Auto-Ignition Temperature & ${ }^{\circ} \mathrm{C}$ & & • & & - & & 217 \\
\hline Flash Point & ${ }^{\circ} \mathrm{C}$ & & • & & $\cdot{ }^{a}$ & & 255 \\
\hline Vapour Pressure ${ }^{k}$ & $\mathrm{mmHg}$ & • & - & & & & 197 \\
\hline Water Solubility & $\mathrm{g} \mathrm{L}^{-1}$ & .1 & & & & $\cdot{ }^{\mathrm{m}}$ & 84 \\
\hline Viscosity $^{n}$ & $\mathrm{cP}$ & & & .0 & & & 218 \\
\hline Enthalpy of Vaporisation ${ }^{p}$ & $\mathrm{~kJ} \mathrm{~L}^{-1 \mathrm{q}}$ & - & & ${ }^{r}$ & & & 208 \\
\hline Heat Capacity ${ }^{\mathrm{s}}$ & $\mathrm{J} \mathrm{L}^{-1} \mathrm{~K}^{-1 \mathrm{t}}$ & - & & . u & & & 200 \\
\hline
\end{tabular}

a) Including ChemSpider (Reference 3). b) At $20^{\circ} \mathrm{C}$. c) Reference 19 . d)Sodium D-Line at $20^{\circ} \mathrm{C}$. e) The data for Cyrene was obtained from reference 20. f) Reference 21. g) Reference 22. h) This is the log hexadecane - air partition coefficient. i) Reference 22. j) Calculated according to the method outlined in reference 23. k) At 20 ${ }^{\circ} \mathrm{C} .1$ ) Data $\log$ transformed for PCA. m) Reference 24. n) At $20^{\circ}$ C. o) Aspen Properties (reference 2). Using either the

19 Abboud J.-L. M.; Notari R., Pure Appl. Chem., 1999, 71, 645-718.

20 Sherwood J.; De bruyn M.; Constantinou A.; Moity L.; McElroy C. R.; Farmer T. J. Duncan T.; Raverty W.; Hunt A. J.; Clark J. H., Chem. Comm., 2014, 50, 9650-9652.

21 Marcus Y., J. Soln. Chem., 1984, 13, 599-624.

22 Abraham M. H., Chem. Soc. Rev., 1993, 73-83.

23 Abraham, M. H.; McGowan, J. C., Chromatographia, 1987, 23, 243-246.

24 Carlson R., Carlson, J. E. Design and Optimization in Organic Synthesis, Elsevier, Amsterdam, 2005. 
NIST/TDE or PDDS9 equations and parameters. p) At normal boiling point. q) Data in $\mathrm{J} \mathrm{mol}^{-1}$ which was used in the PCA, however, data displayed in $\mathrm{kJ} \mathrm{L}^{-1}$ as this is a more appropriate set of scale up units. r) Aspen Properties (reference 2). TDE Watson equation and parameters. s) Constant pressure heat capacity at $20^{\circ} \mathrm{C}$. $\mathrm{t}$ ) Data in $\mathrm{J} \mathrm{mol}^{-1}$ which was used in the PCA, however, data displayed in $\mathrm{kJ} \mathrm{L}^{-1}$ as this is a more appropriate set of scale up units. $\mathrm{u}$ ) Aspen Properties (reference 2), CPLDECS or CPLTPO equation and parameters. 


\section{Physical Properties Summary Charts}
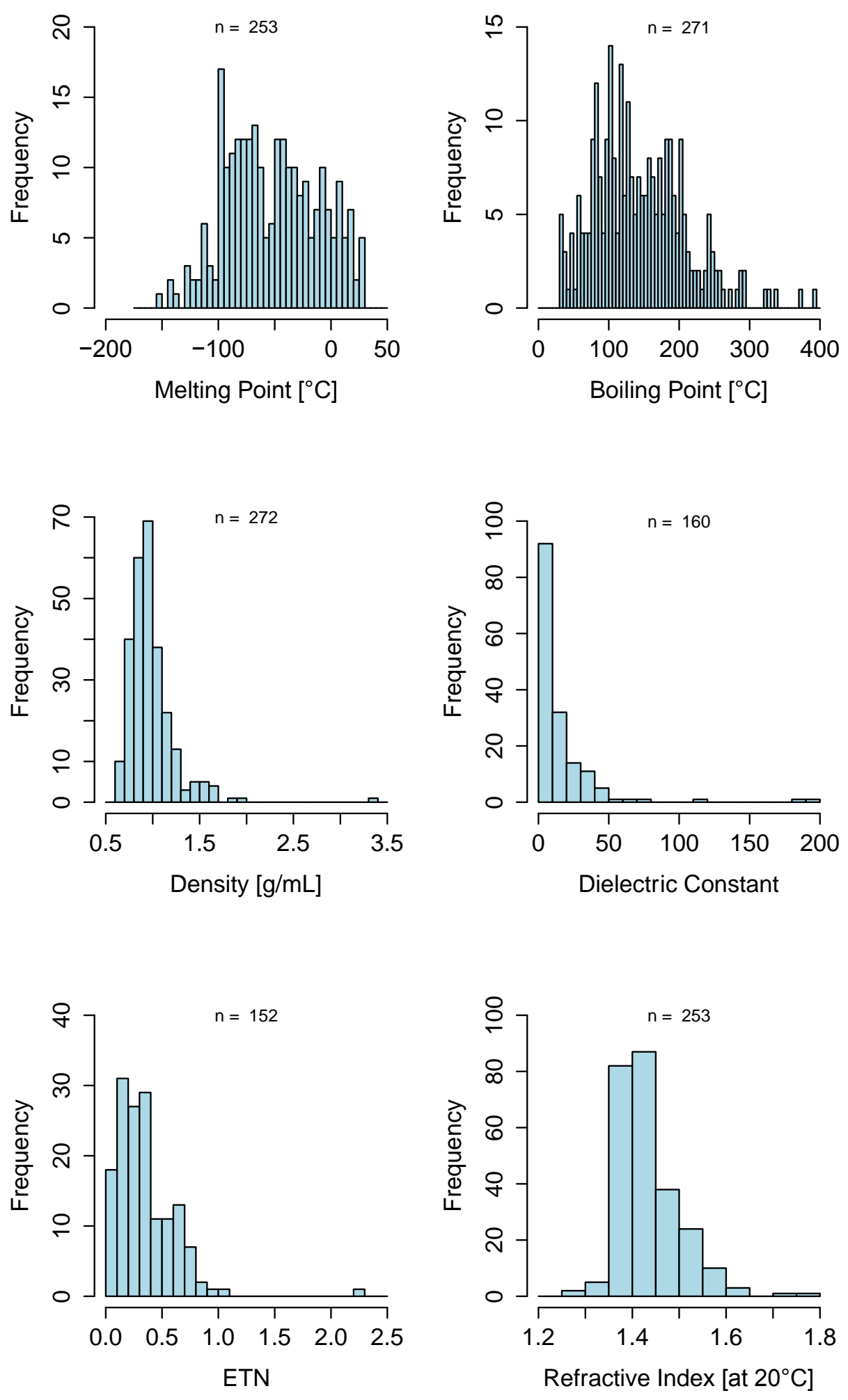

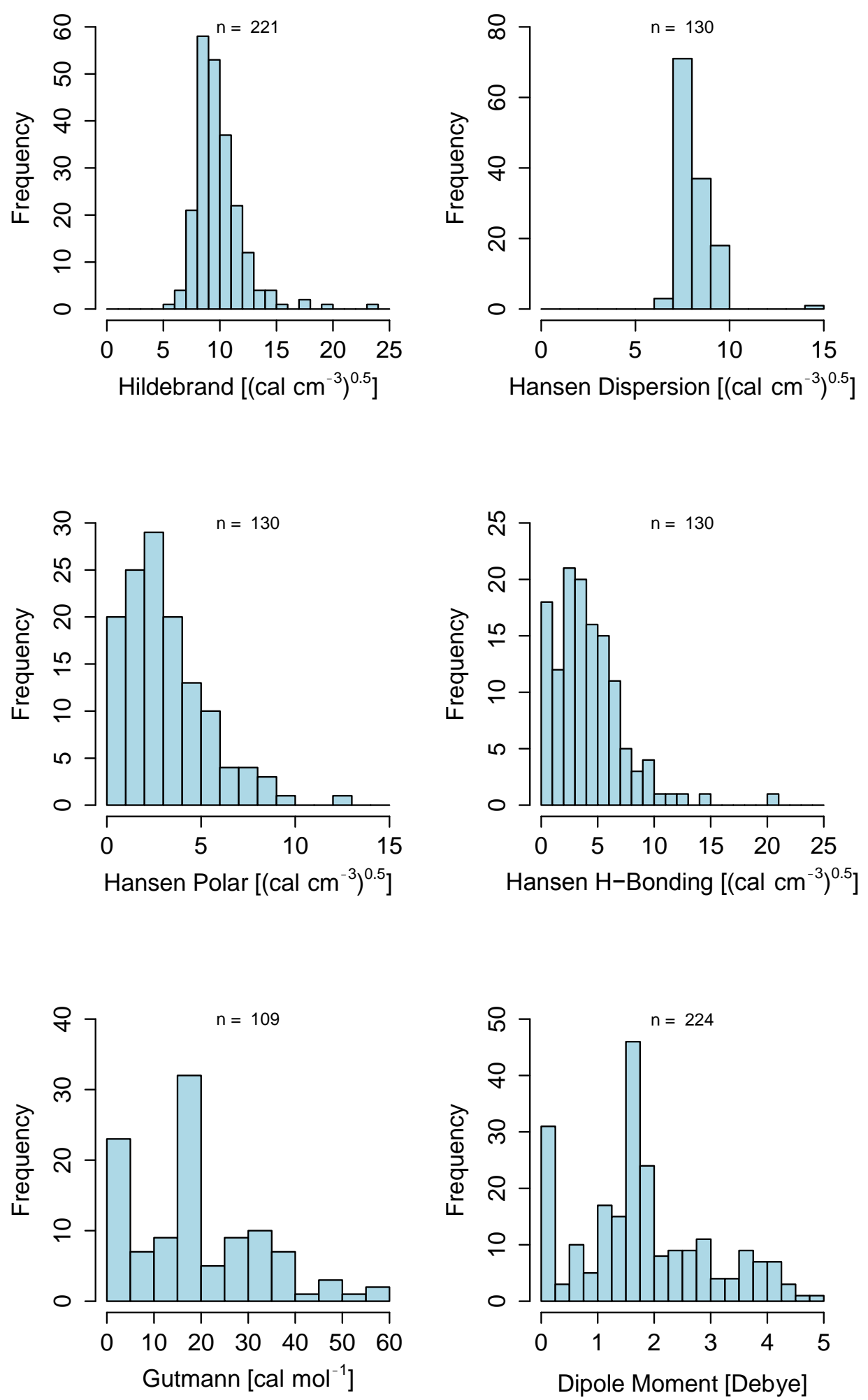

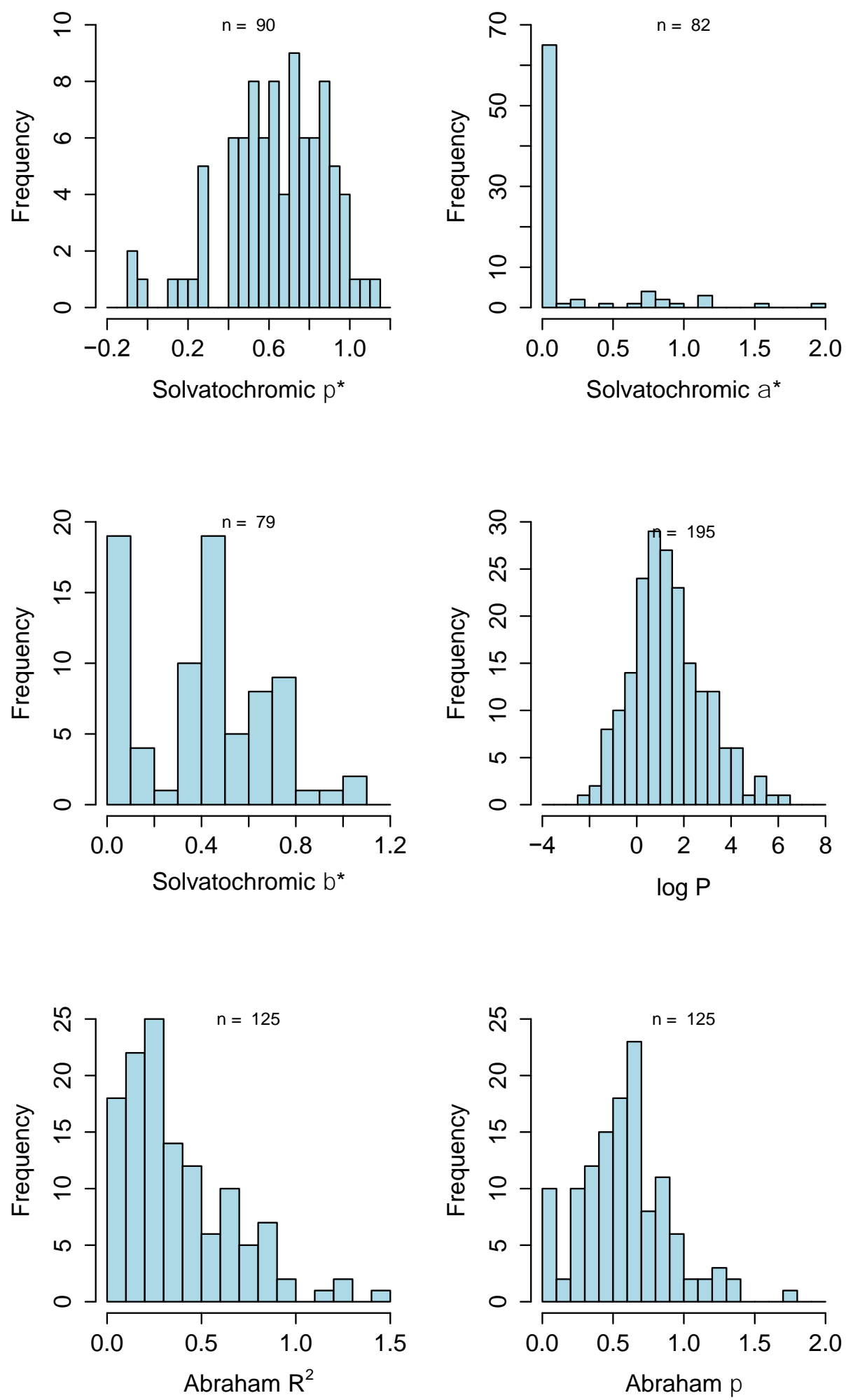

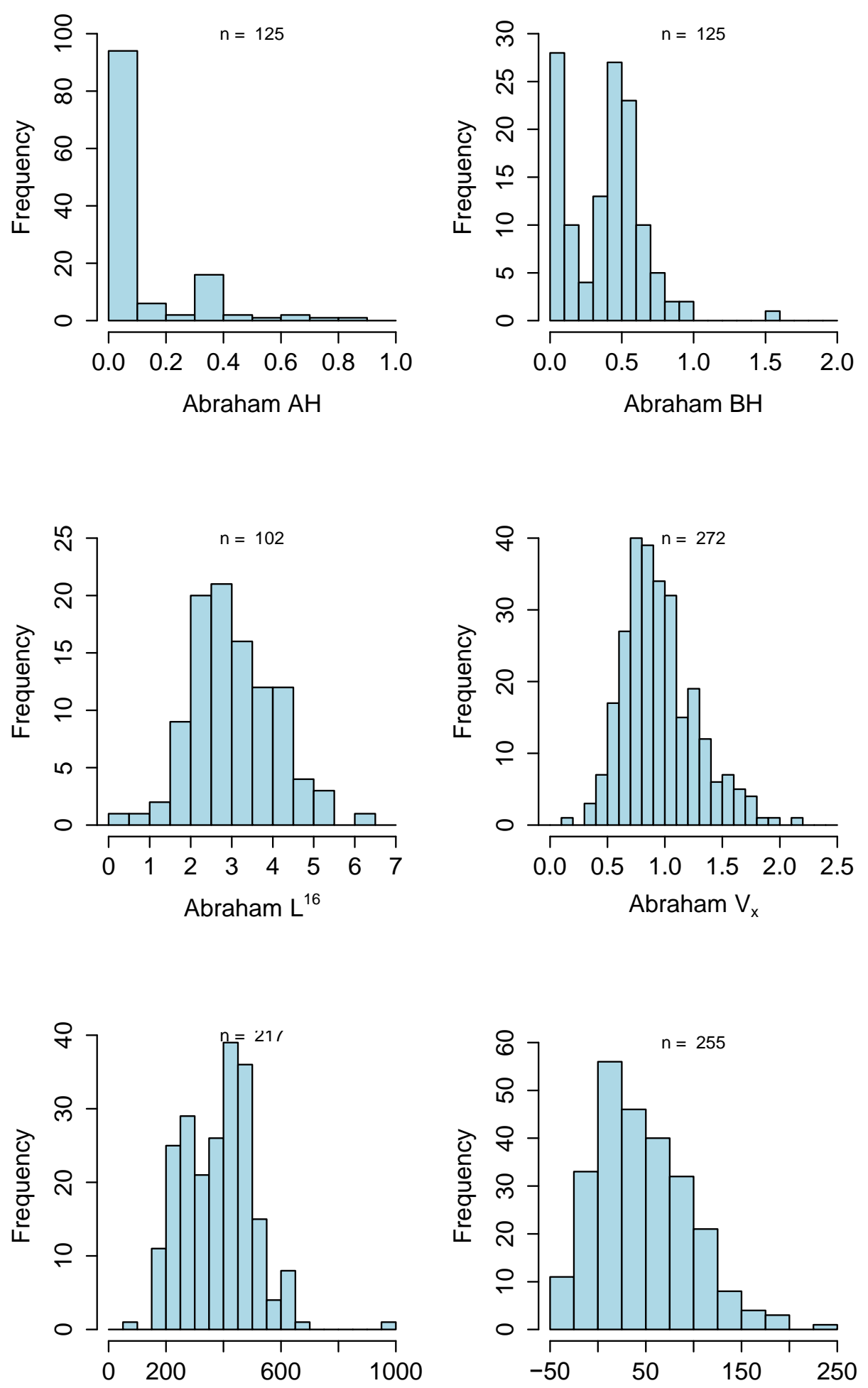

Auto-Ignition Temperature $\left[{ }^{\circ} \mathrm{C}\right]$

Flash Point Temperature $\left[{ }^{\circ} \mathrm{C}\right]$ 

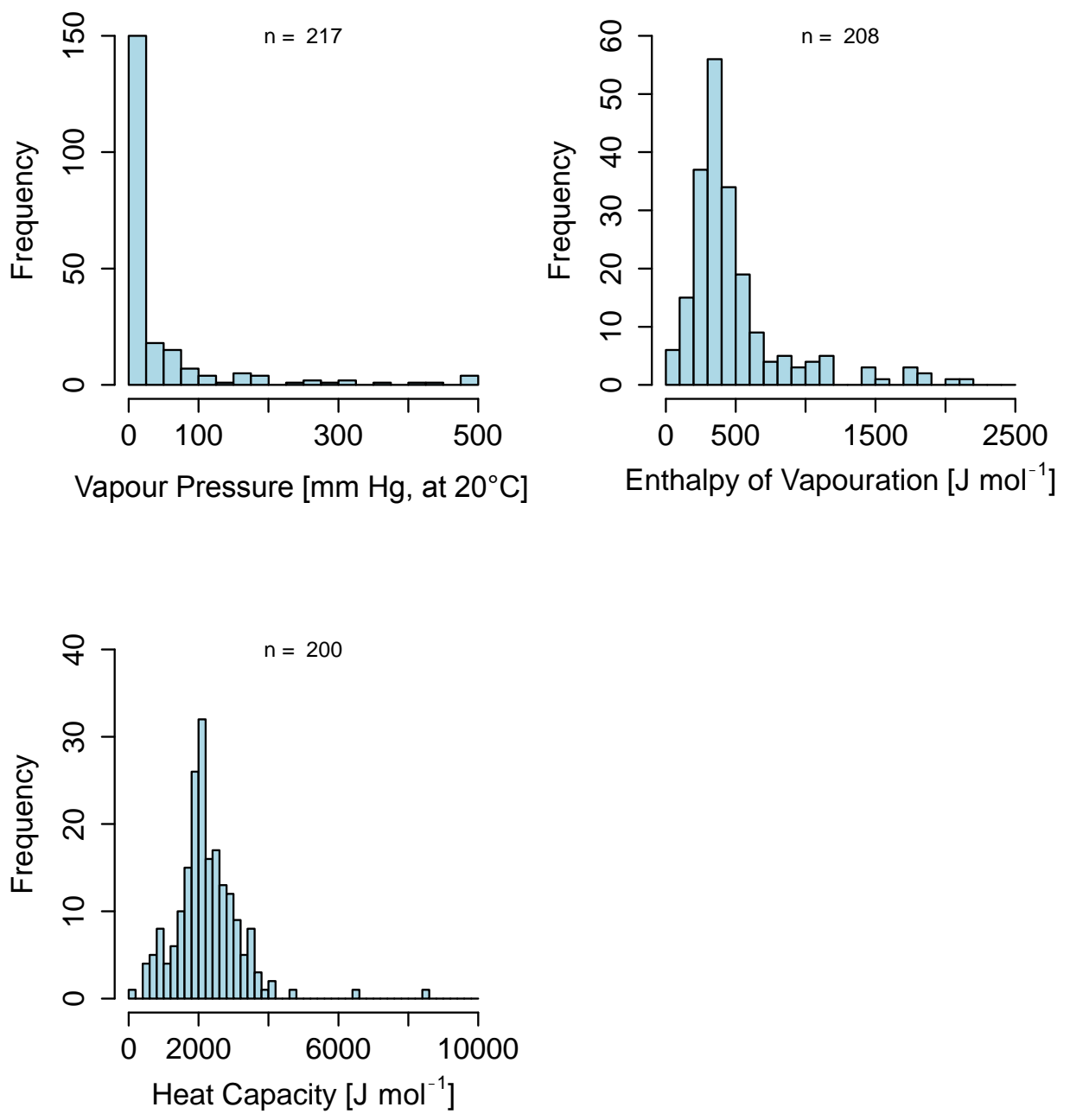


\section{Computed Properties}

\subsection{Informatics}

A master list of SMILES strings was created for all of the solvents in the database. The OpenBabel ${ }^{25}$ suite was used to generate the corresponding InChI and InChIKeys ${ }^{26,27}$ and the initial 3D structures in SDF format ${ }^{28,29}$ for the conformation searches in Spartan.

The obprop module was used to generate the corresponding molecular formula and count the number of bonds between the atoms. This information was used to calculate McGowan $V_{X}$ parameter, ${ }^{30}$ divided by 100 .

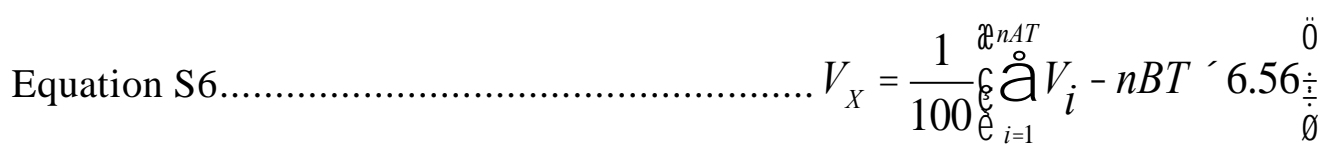

Where; $V_{i}$ is the atom volume parameter of the element (see below), the summation runs over all of the atoms $(n A T), n B T$ is the number of bonds in the molecule (no distinction made between single, double or triple bonds).

The atomic volume parameters used in the calculation are: Carbon - 16.35, Hydrogen - 8.71, Bromine - 26.21, Chlorine - 20.95, Fluorine - 10.48, Iodine - 34.53, Nitrogen 14.39, Oxygen - 12.43, Phosphorous - 24 and Sulfur -22.91.

\subsection{Quantum Mechanical (Gaussian).}

Conformer searches were preformed using Spartan'04, ${ }^{31}$ using the Systematic/MonteCarlo method with MMFF94 ${ }^{32}$ geometry optimisation and a $50 \mathrm{~kJ} \mathrm{~mol}^{-1}(11.95 \mathrm{kcal}$ $\mathrm{mol}^{-1}$ ) search window. Up to 20 of the lowest energy conformers where selected for further optimisation and property calculation using Density Functional Theory (DFT).

DFT calculations were performed in the Gaussian 09 suite. ${ }^{33}$ The geometries were optimised using B3LYP functional. ${ }^{34}$ For the elements hydrogen, nitrogen, carbon,

25 a) O'Boyle N. M.; Banck M.; James C. A.; Morley C.; Vandermeersch T.; G. R. Hutchison G.

R. Journal of Cheminformatics, 2011, 3:33. b) www.openbabel.org.

26 Heller S.; McNaught A.; Stein S.; Tchekhovskoi D.; Pletnev I. J Cheminform. 2013, 5:7.

27 From the command line: babel Solvents.smi Solvents.inchi - xK

Dalby A.; Nourse J. G.; Hounshell W. D.; Gushurst A. K. I.; Grier D. L.; Leland B. A.; Laufer J. J. Chem. Inf. Comput. Sci., 1992, 32, 244-255.

From the command line: babel Solvents.smi Solvents.sdf --gen3D

Abraham M. H.; McGowan J. C. Chromatographia, 1987, 23, 243-246.

Spartan'04, Wavefunction, Inc., Irvine, CA. The calculation methods, except for molecular mechanics and semi-empirical models have been documented in: Kong J.; White C. A.; Krylov A. I.; Sherrill C. D.; Adamson R. D.; Furlani T. R.; Lee M. S.; Gwaltney S. R.; Adams T. R.; Ochsenfeld C.; Gilbert A. T. B.; Kedziora G. S.; Rassolov V. A.; Maurice D. R.; Nair N.; Shao Y.; Besley N. A.; Maslen P. E.; Dombroski J. P.; Dashel H.; Zhang W.; Korambath P. P.; Baker J.; Byrd E. F. C.; Van Voorhis T.; Oumi M.; Hirata S.; Hsu C.-P.; Ishikawa N.; Florian J.; Warshel A.; Johnson B. G.; Gill P. M. W.; Head-Gordon M.; Pople J. A. J. Computational Chem., 2000, 21, 1532-1548. 
oxygen, fluorine, silicon, phosphorous, sulfur, chlorine and bromine the $6-31+\mathrm{G}(\mathrm{d})^{35}$ basis state was used and the LANL2DZ ${ }^{36}$ basis set for iodine. Frequency calculations ${ }^{37}$ were performed using the harmonic oscillator - rigid rotor approximation to confirm that each geometry was at a stationary point (no imaginary frequencies) upon the potential energy surface. Thermochemical values were obtained, without scaling, for a standard state of $298.15 \mathrm{~K}$ and $1 \mathrm{~atm}$. [\# P opt freq=noraman b3lyp/gen pseudo=read nosymm].

Three single point energy calculations, upon the optimised geometries, at the same level of theory, were performed for the neutral $(\mathrm{q}=0, \mathrm{~m}=1)$, radical cation $(\mathrm{q}=1, \mathrm{~m}$ $=2)$ and radical anion $(\mathrm{q}=-1, \mathrm{~m}=2)$ species. These calculations used the restricted / restricted open-shell formulism with a 'tight' self-consistent field (SCF) convergence criteria.

$$
\begin{array}{lll}
\text { rb3 lyp } / 6-31+G(d) & \text { scf=tight nosymm } & \text { For Neutral Species. } \\
\text { rob3lyp } / 6-31+G(d) & \text { scf=tight nosymm } & \text { For Radical Species. }
\end{array}
$$

The vertical ionisation potential (IP) was calculated by the $\triangle \mathrm{SCF}$ method $^{38}$ according to:

Nakatsuji, H.; Caricato, M.; Li, X.; Hratchian, H. P.; Izmaylov, A. F.; Bloino, J.; Zheng, G.; Sonnenberg, J. L.; Hada, M.; Ehara, M.; Toyota, K.; Fukuda, R.; Hasegawa, J.; Ishida, M.; Nakajima, T.; Honda, Y.; Kitao, O.; Nakai, H.; Vreven, T.; Montgomery, J. A., Jr.; Peralta, J. E.; Ogliaro, F.; Bearpark, M.; Heyd, J. J.; Brothers, E.; Kudin, K. N.; Staroverov, V. N.; Kobayashi, R.; Normand, J.; Raghavachari, K.; Rendell, A.; Burant, J. C.; Iyengar, S. S.; Tomasi, J.; Cossi, M.; Rega, N.; Millam, N. J.; Klene, M.; Knox, J. E.; Cross, J. B.; Bakken, V.; Adamo, C.; Jaramillo, J.; Gomperts, R.; Stratmann, R. E.; Yazyev, O.; Austin, A. J.; Cammi, R.; Pomelli, C.; Ochterski, J. W.; Martin, R. L.; Morokuma, K.; Zakrzewski, V. G.; Voth, G. A.; Salvador, P.; Dannenberg, J. J.; Dapprich, S.; Daniels, A. D.; Farkas, Ö.; Foresman, J. B.; Ortiz, J. V.; Cioslowski, J.; Fox, D. J. Gaussian, Inc., Wallingford CT, 2009.

a) Becke A. D. J. Chem. Phys., 1993, 98, 5648-52. b) Lee C.; Yang W.; Parr R. G. Phys. Rev. B, 1988, 37, 785-789. c) Miehlich B.; Savin A.; Stoll H.; Preuss H. Chem. Phys. Lett., 1989, 157, 200-206. d) Vosko S. H.; Wilk L.; Nusair M. Can. J. Phys. 1980, 58, 1200-1211. e) Stephens P. J.; Devlin F. J.; Chabalowski C. F.; Frisch M. J. J. Phys. Chem. 1994, 98, 11623 11627.

a) Ditchfield R.; Hehre W. J.; Pople J. A. J. Chem. Phys., 1971, 54, 724-728. b) Hehre W. J.; Ditchfield R.; Pople J. A. J. Chem. Phys., 1972, 56, 2257-2261. c) Hariharan P. C.; Pople J. A. Theor. Chem. Acc., 1973, 28, 213-222. d) Hariharan P. C.; Pople J. A. Mol. Phys., 1974, 27, 209-14. e) Gordon M. S. Chem. Phys. Lett., 1980, 76, 163-168. f) Francl M. M.; Pietro W. J.; Hehre W. J.; Binkley J. S.; DeFrees D. J.; Pople J. A.; Gordon M. S. J. Chem. Phys., 1982, 77, 3654-3665. h) Binning Jr. R. C.; Curtiss L. A. J. Comp. Chem., 1990, 11, 12061216. i) Blaudeau J.-P.; McGrath M. P.; Curtiss L. A.; Radom L. J. Chem. Phys., 1997, 107, 5016-21. j) Rassolov V. A.; Pople J. A.; Ratner M. A.; Windus T. L. J. Chem. Phys., 1998, 109, 1223-1229. k) Rassolov V. A.; Ratner M. A.; Pople J. A.; Redfern P. C.; Curtiss L. A. J. Comp. Chem., 2001, 22, 976-984. 1) Clark T.; Chandrasekhar J.; Spitznagel G. W.; Schleyer P. v. R. J. Comp. Chem., 1983, 4, 294-301. m) Frisch M. J.; Pople J. A.; Binkley J. S. J. Chem. Phys., 1984, 80, 3265-3269.

a) Dunning Jr. T. H.; Hay P. J. Modern Theoretical Chemistry, Ed. H. F. Schaefer III, Vol. 3, Plenum, New York, 1977, 1-28. b) Hay P. J.; Wadt W. R. J. Chem. Phys., 1985, 82, 270-283. c) Wadt W. R.; Hay P. J. J. Chem. Phys., 1985, 82, 284-298. d) Hay P. J.; Wadt W. R. J. Chem. Phys., 1985, 82, 299-310.

The noraman option was selected to reduce the frequency calculation time. The Gaussian manual states that there is a 10 to $30 \%$ overhead for calculating the Raman intensities, which are unnecessary to confirm that the geometry is at a stationary point. 
Equation S7.

$I P=E_{M^{\cdot+}}-E_{M}$

Similarly, the electron affinity $(E A)$ was calculated by:

Equation S8. $E A=E_{M^{-}}-E_{M}$

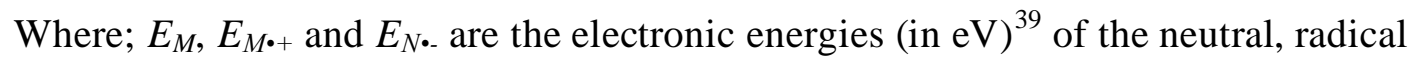
cation and radical anion species.

From conceptual DFT, ${ }^{40}$ the chemical potential $(\mu),{ }^{41}$ hardness $(\eta)^{42}$ and the electrophilicity index $(\omega)^{43}$ are calculated (in eV) by:

Equation $\mathrm{S} 9$

$=\frac{1}{2}(I P+E A)$

Equation S10

$=I P \quad E A$

Equation S11

$=\frac{2}{2}$

The polarisability $(\alpha)$ and hyperpolarisability $(\beta)$ was calculated under the static (constant, $\alpha_{0}$ and $\left.\beta_{0}\right)$ and dynamic $\left(\alpha_{598 \mathrm{~nm}}\right.$ and $\left.\beta_{598 \mathrm{~nm}}\right)$ electric field regimes. A wavelength of $598 \mathrm{~nm}$ was chosen on the basis that the refractive index of a compound is commonly measured using the Fraunhofer sodium emission D-lines $\left(\mathrm{D}_{1}\right.$ $=589.592$ and $\mathrm{D}_{2}=588.995 \mathrm{~nm}$ ).

For the calculation of molecular polarisabilities, it has been shown that diffuse functionals in the basis set are important, ${ }^{44,45}$ and the B3LYP functional gives acceptable results when compared to $\operatorname{CCSD}(\mathrm{T})$ and experimental results. ${ }^{46,47}$ The polarisabilities were calculated using the $6-31++\mathrm{G}(\mathrm{d}, \mathrm{p})$ basis set was used for the elements hydrogen, nitrogen, carbon, oxygen, fluorine, silicon, phosphorous, sulfur, chlorine and bromine and the LANL2DZ basis set for iodine. [\#p freq=raman cphf=rdfreq b3lyp/gen pseudo=read nosymm polar].

The polarisability to an electric field is given by the trace of the polarisability tensor.

39

The conversion from atomic units (au) to $\mathrm{eV}$ was achieved by multiplying by the 2010 CODATA recommended value of 27.21138505. Mohr P. J.; Taylor B. N.; Newell D. B. Reviews of Modern Physics, 2012, 84, 1527-1605.

40 Geerlings P.; De Proft F.; Langenaeker W. Chem. Rev. 2003, 103, 1793-1874.

Parr R. G.; Donnelly R. A.; Levy M. ; Palke W. E. J. Chem. Phys. 1978, 68, 3801.

Parr R. G.; Pearson R. G. J. Am. Chem. Soc. 1983, 105, 7512-7516.

Parr R. G.; Von Szentpaly L.; Liu S. B. J. Am. Chem. Soc., 1999, 121, 1922-1924.

"A mean-field approach for the determination of the polarizibilities for the water molecule in liquid state", Gubskaya A. V.; Kusalik P. G. Computational Aspects of Electric Polarizability Calculations: Atoms, Molecules and Clusters, Ed. G. Maroulis, IOS Press, 2006.

Vila F. D.; Strubbe D. A.; Takimoto Y.; Andrade X.; Rubio A.; Louie S. G.; Rehr J. J. J. Chem. Phys., 2010, 133, 034111.

de Souza F. A. L.; Jorge F. E., J. Braz. Chem. Soc., 2013, 24 1357-1365.

Ando S. J. Photopolymer Sci. Tech., 2006, 19, 351-360. 
Equation S12

$$
=\frac{x x+y y+z z}{3}
$$

The anisotropy of polarisability $(\Delta \alpha)$ can be calculated by;

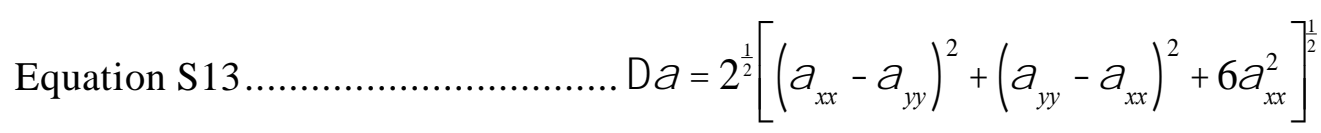

The hyperpolarisibility $\beta$ is calculated:

$$
\begin{aligned}
& =\left(\begin{array}{l}
2 \\
x
\end{array} \quad \begin{array}{l}
2 \\
y
\end{array}+\begin{array}{l}
2 \\
z
\end{array}\right)
\end{aligned}
$$

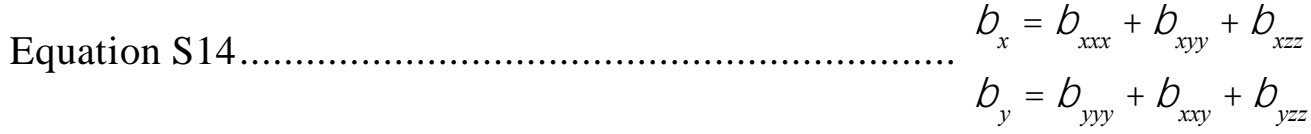

$$
\begin{aligned}
& { }_{x}={ }_{z z z}+{ }_{x y z}+{ }_{y y z}
\end{aligned}
$$

Gaussian reports the $\alpha$ and $\beta$ tensor values in atomic units (au). These can be converted to the corresponding electrostatic units by multiplying by $0.1482 \times 10^{-12}$ and $8.6393 \times 10^{-33}$ respectively.

The dipole moment (debyes) of the neutral species was also extracted.

The Boltzmann weighted average properties mentioned above, were calculated for the conformational ensemble ${ }^{48}$ for each compound using:

Equation S15

$$
\bar{P}=\frac{{ }_{i=1}^{N} P_{i} \exp \frac{G_{i}}{R T} \div}{{ }_{i=1}^{N} \exp \frac{G_{i}}{R T} \div}
$$

Where; $P_{i}$ is the property value of conformer $i, N$ is the number of conformers in the ensemble, $G_{i}$ is the Gibbs Free Energy $\left(\mathrm{kJ} \mathrm{mol}^{-1}\right)^{49}$ of the $i^{\text {th }}$ conformer, $R$ is the Universal Gas Constant $\left(8.3144621 \mathrm{~J} \mathrm{~K}^{-1} \mathrm{~mol}^{-1}\right)^{50}$ and $T$ is the absolute temperature $(K)$.

\section{3 cosmotherm}

The $\sigma$-profiles of solvent molecules that weren't already present in the COSMOtherm $^{51}$ database were generated using COSMOconf. ${ }^{52}$ The built in BPTZVPD-Fine-COSMO + Gas method was used without any modification of the

\footnotetext{
$48 \quad$ Lavy T.; Harries D.; Goldblum A. J. Phys. Chem. A, 2011, 115, 5794-5809.

49 The conversion from atomic units (au) to $\mathrm{kJ} \mathrm{mol}^{-1}$ was achieved by multiplying by the 2010 CODATA recommended value of 2625.49962 (see reference 39).

50 The 2010 CODATA recommended value (see reference 39 ).

51 a) Eckert F.; Klamt A. COSMOtherm, version C3.0, Release 14.01; COSMOlogic, GmbH \& Co. KG, Leverkusen, Germany, 2013. b) Eckert F.; Klamt A. AIChE Journal, 2002, 48, 369 385 .

52 Diedenhofen M.; Eckert F.; Hellweg A.; Huniar U.; Loschen C.; Klamt A.; Pohler L.; Reinisch J.; Schroer A.; Steffen C.; Thomas K.; Wichmann K. COSMOconX, version 3.0, COSMOlogic, GmbH \& Co. KG, Imbacher Weg 46, 51379 Leverkusen, Germany.
} 
default parameters. The method comprises broadly of the following steps: Balloon $^{53}$ conformer search generates a list of conformers. After geometric clustering, up to 150 of the lowest energy are subjected to COSMO/BP/SV(P) ${ }^{54,55,56}$ single point calculations using Turbomole. ${ }^{57} \mathrm{Up}$ to 50 conformers within a $25 \mathrm{kcal} \mathrm{mol}^{-1}$ window were optimised at the same level of theory using a 'loose' convergence threshold. After geometric and chemical potential clustering, up to 12 conformers within a 10 $\mathrm{kcal} \mathrm{mol}^{-1}$ window were optimised at the COSMO/BP/TZVP ${ }^{58}$ level of theory. Upon resorting and clustering, up to 10 conformers, within a $6 \mathrm{kcal} \mathrm{mol}^{-1}$ window, were subjected to COSMO/BP-TZVPD-FINE single point calculation and BP/TZVP optimisation and BP/TZVPD-FINE single point calculations in the gas phase. The extracted geometries, energies and COSMO profiles are imported COSMOtherm.

The COSMOtherm program is based upon the COSMO-RS methodology has been described in detail by Klamt and coworkers. ${ }^{59}$ Briefly, the DFT calculations are performed within a cavity of a polarisable-continuum dielectric medium (COnductorlike Screening MOdel - COSMO). The inner surface of this cavity reacts to the surface charges of the molecule of interest to give a complimentary charge profile. This complex three-dimensional surface is reduced to a two dimensional histogram of charge density, the $\sigma$-profile. A statistical mechanics approach considers all of the electrostatic and hydrogen-bonding interactions in a liquid system as contact interactions of the molecular surfaces of the solvent and solute molecules. The chemical potential of these pair-wise interacting surface element contacts can be calculated, leading to a description of the chemical potential of the whole system under consideration. With this information a range of physical properties can be calculated; vapour-liquid, liquid-liquid and solid-liquid equilibria, hence leading to practically useful predictions for solubilities, partitioning behaviour between immiscible solvents, amongst others.

The following properties were calculated with COSMOtherm (Using the 2014 TZVPD-FINE parameters):

- $\log \mathrm{P}_{\text {ow }}$ (Octanol-Water partition coefficient)

- $\log \mathrm{P}_{\mathrm{hw}}$ (Hexadecane-Water partition coefficient)

- $\log \mathrm{P}_{\mathrm{cw}}$ (Chloroform-Water partition coefficient)

- $\log \mathrm{S}_{\mathrm{S}}^{\mathrm{W}}$ (Solubility of solvent in water, $\mathrm{g} / \mathrm{g}$ )

- $\log S_{\mathrm{W}}^{\mathrm{S}}$ (Solubility of water in solvent, $\mathrm{g} / \mathrm{g}$ )

53 Vainio M. J.; Johnson M. S. J. Chem. Inform and Modelling, 2007, 47, 2462-2474. The MMFF94 (reference 32) force field is used for the geometry optimisation.

$54 \quad$ Klamt A.; Schüürmann G. J. Chem. Soc. Perkin Trans. 2, 1993, 5, 799-805.

55 a) Dirac P. A. M. Proc. Royal Soc. (London) A, 1929, 123, 714-733. b) Slater J. C. Phys. Rev., 1951, 81, 385-390. c) Becke A. D. Phys. Rev. A, 1988, 38, 3098-3100. d) Perdew J. P. Phys. Rev. $B, \mathbf{1 9 8 6}, 33,8822-8824$. e) Reference 34d.

Schäfer A.; Horn H.; Ahlrichs R. J. Chem. Phys., 1992, 97, 2571.

57 TURBOMOLE V6.4 2012, a development of University of Karlsruhe and Forschungszentrum Karlsruhe GmbH, 1989-2007, TURBOMOLE GmbH, since 2007; available from http://www.turbomole.com.

$58 \quad$ Schäfer A.; Huber C.; Ahlrichs R. J. Chem. Phys., 1994, 100, 5829.

59 Klamt A. COSMO-RS: From quantum Chemistry to Fluid Phase Thermodynamics and Drug Design. Elsevier: Amsterdam, 2005. 
- $\sigma$-Moments $1-6$

- Hydrogen-Bonding Donor $1-4$.

- Hydrogen-Bonding Acceptor $1-4$.

- Surface area $\left(\AA^{2}\right)$.

- Molecular volume $\left(\AA^{3}\right)$.

- Dipole moment (debyes)

The QSPR model of the Abraham linear free-energy descriptors ${ }^{60}$ for each solvent was calculated using the 2014 TZVP parameter set.

- Abraham A (Acidity)

- Abraham B (Basicity)

- Abraham S (Polarity/Polarisability)

- Abraham E (Excess molar refraction)

- Abraham V (Hydrophobicity)

\subsection{Chiral Molecules}

With some notable exceptions, solvents that have one or more chiral centres, it has been assumed that the manufacturing process has produced all stereoisomers in equimolar proportions. On the basis that enantiomers have identical properties, which the exception to their behaviour towards polarised light, only single enantiomers have been considered during the modelling process. Thus for compounds which have multiple chiral centres, all racemic distereoisomers have been explicitly modelled.

For the Gaussian based calculations, the physical properties of each diastereoisomer conformer ensemble, normalised to each diastereomers lowest energy conformer, are Boltzmann weighted as described above. The final physical properties of the bulk properties are assumed to be an average of these weighted ensembles.

\subsection{Derived Descriptors}

The way PCA works is that it 'looks' for linear, first order, relationships between descriptors/physical properties in the data set. However, some of the computed descriptors do not have a direct linear relationship with the physical properties, instead they are either a combination of descriptors or have a power relationship. In order to create the linear relationships required for the analysis, some of the descriptors/properties have had mathematical transformations applied to them, based upon the underlying physical relationships between the parameters.

A case in point is that the refractive index of a solvent. The Lorentz-Lorenz equation (Equation $\mathrm{S} 1$ ) relates the square of the refractive index, $n$, to the polarisibility, $\alpha$, times the number of molecules per unit volume (density). Rearranging the LorentzLorenz equation gives Equation S16, which now a linear relationship between the polarisability, and the refractive index and molar volume. ${ }^{61}$

60 Zissimos A. M.; Abraham M. H.; Klamt A.; Eckert F.; Wood J. J. Chem. Inf. Comput. Sci., 2002, 42, 1320-1331.

61 Physical and unit conversion constants have been ignored, as it is the correlation between the physical properties and descriptors that is key for the PCA. 


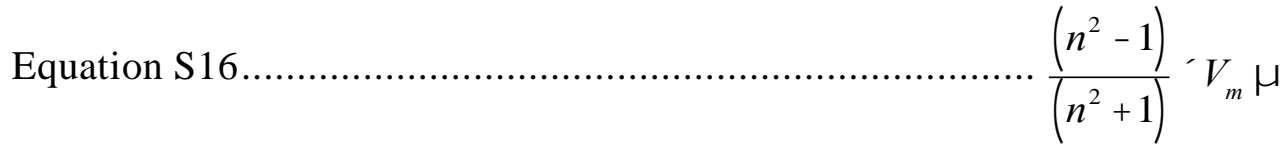

The experiment molar volume $\left(V_{m}\right)$ is not available for all solvents; therefore it has been derived from the molecular weight and density. Comparing the derived $V_{m}$ versus the available 230 experimental values shows that vast majority of the falls with $\pm 2 \%$, with only a few outliers and more than $50 \%$ of the solvents had values within $\pm 0.1 \%$.

Any equilibrium constants (partition, solubility, etc) were used in their $\log _{10}$ form (c.f. Equilibrium Isomer Equation $\Delta G=-R T \ln \left[K_{a}\right]$ ) so that the phenomenon is transformed back into a linear energy relationship. 


\section{Calculated Properties Summary Charts}
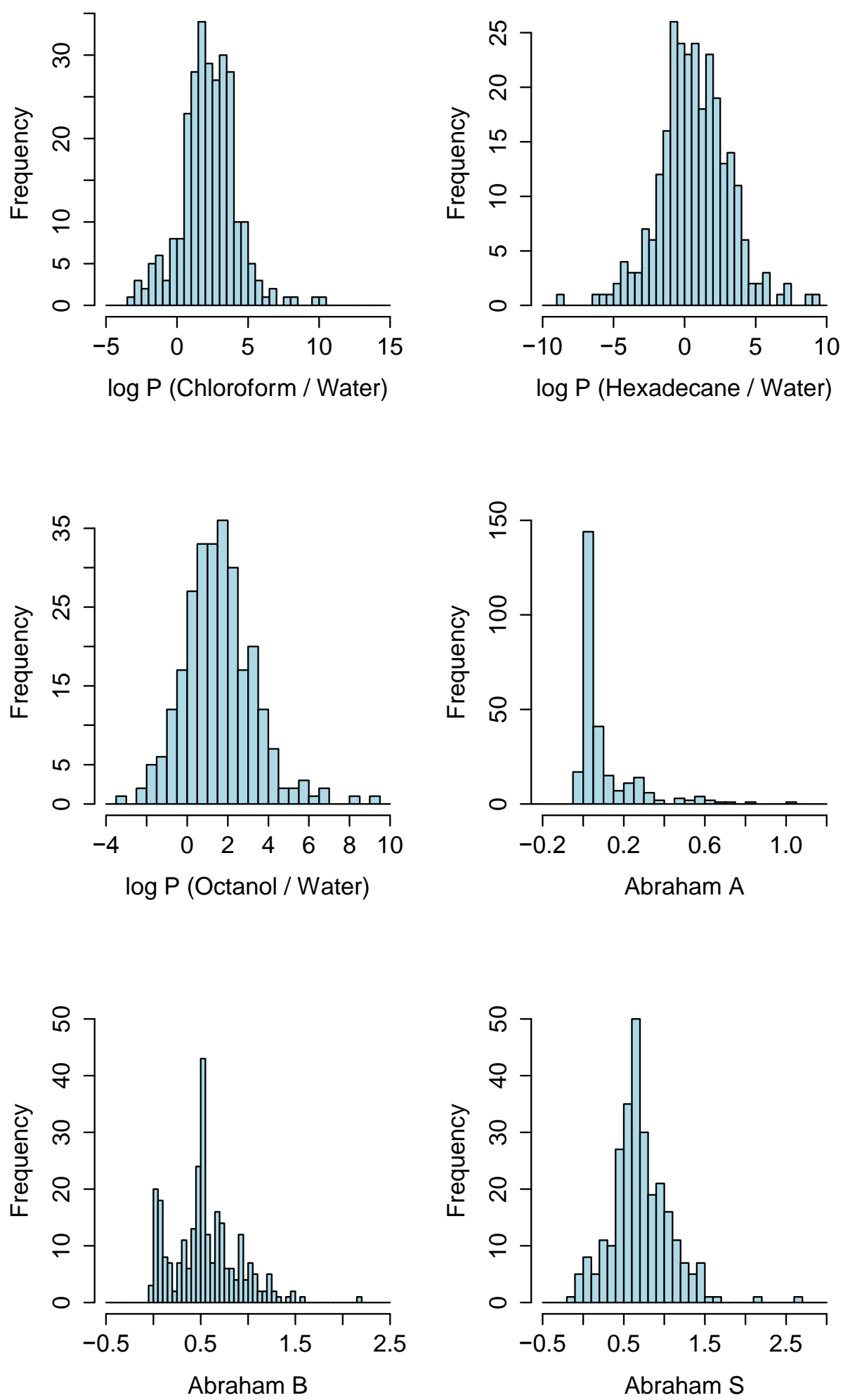

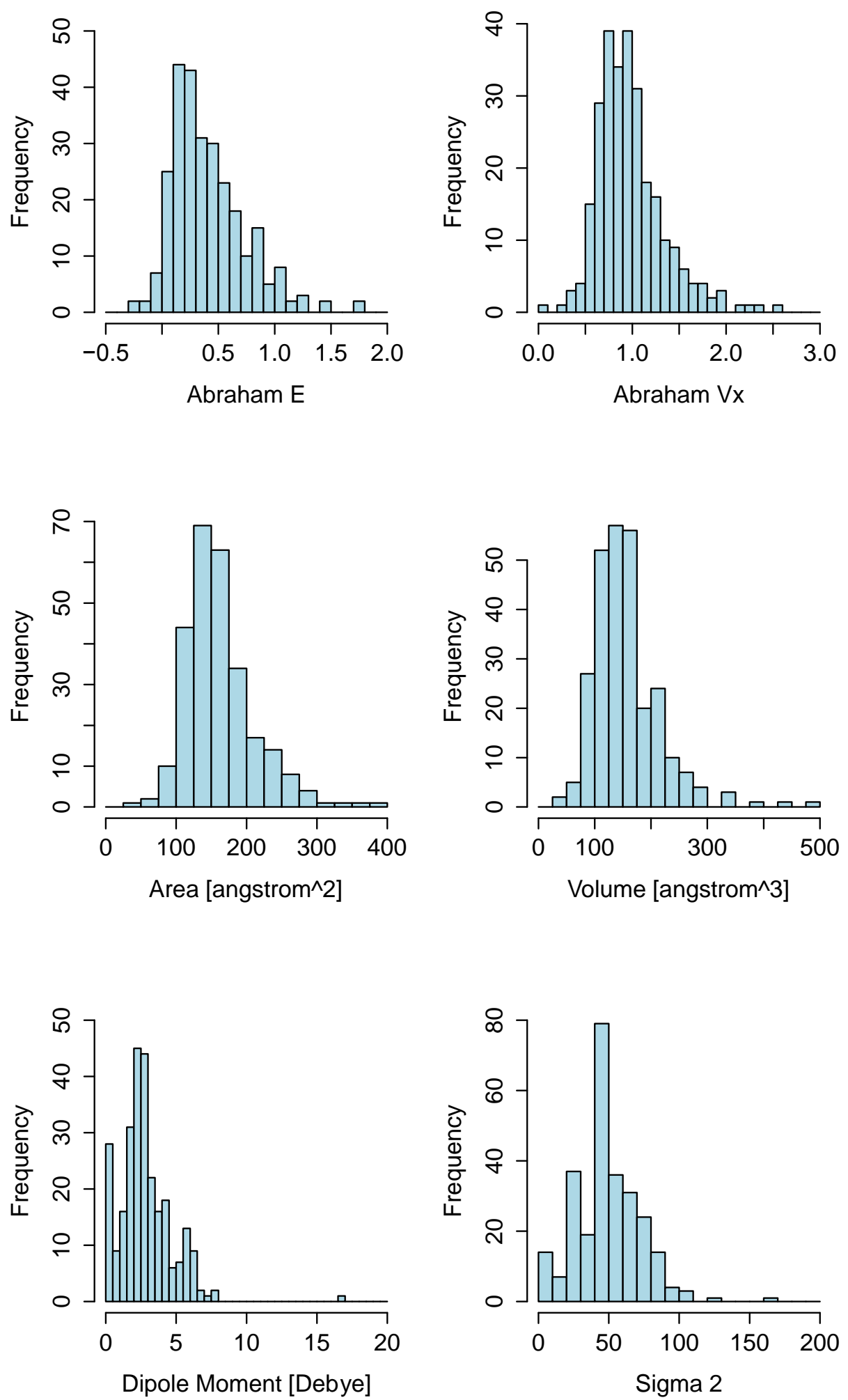

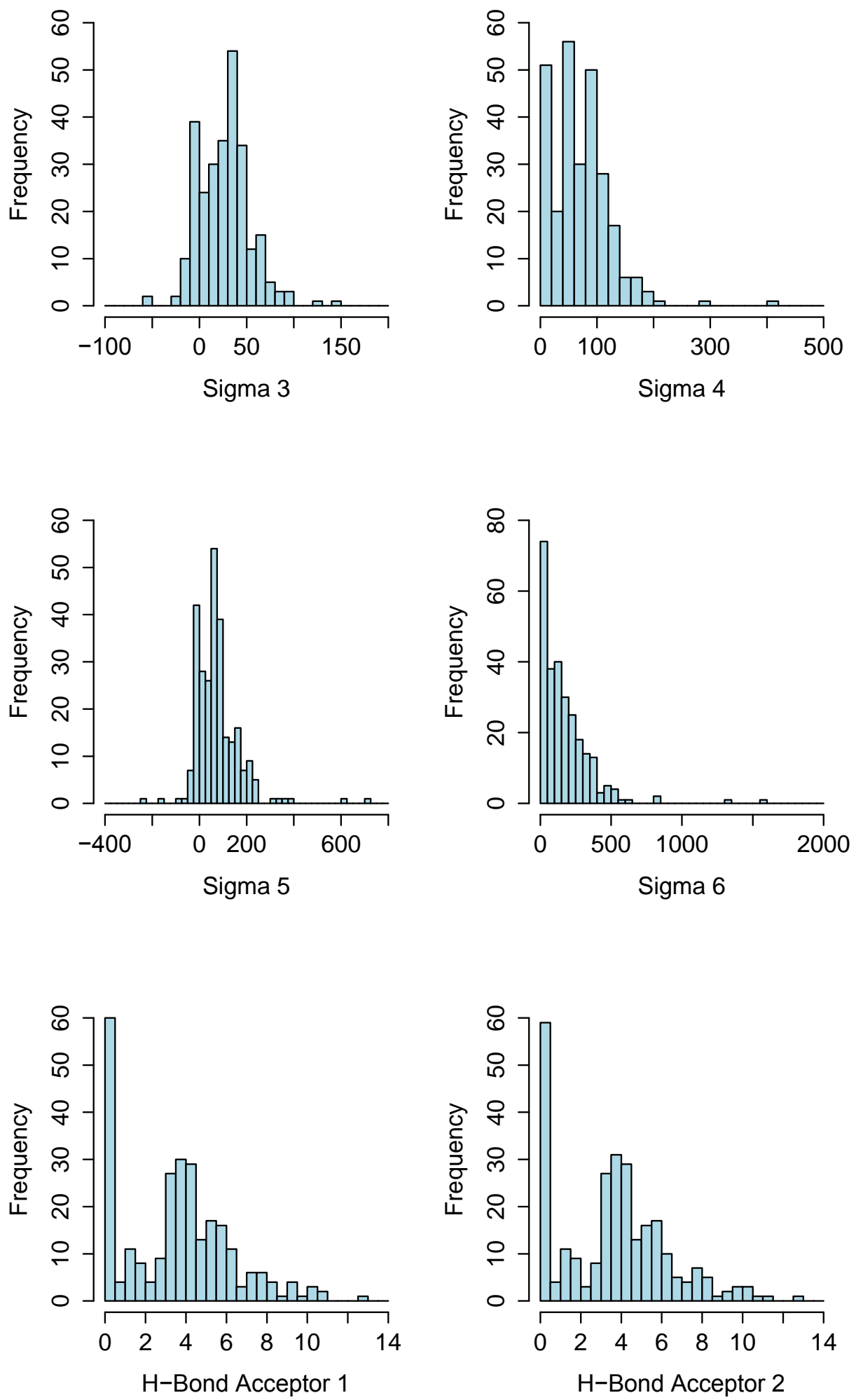

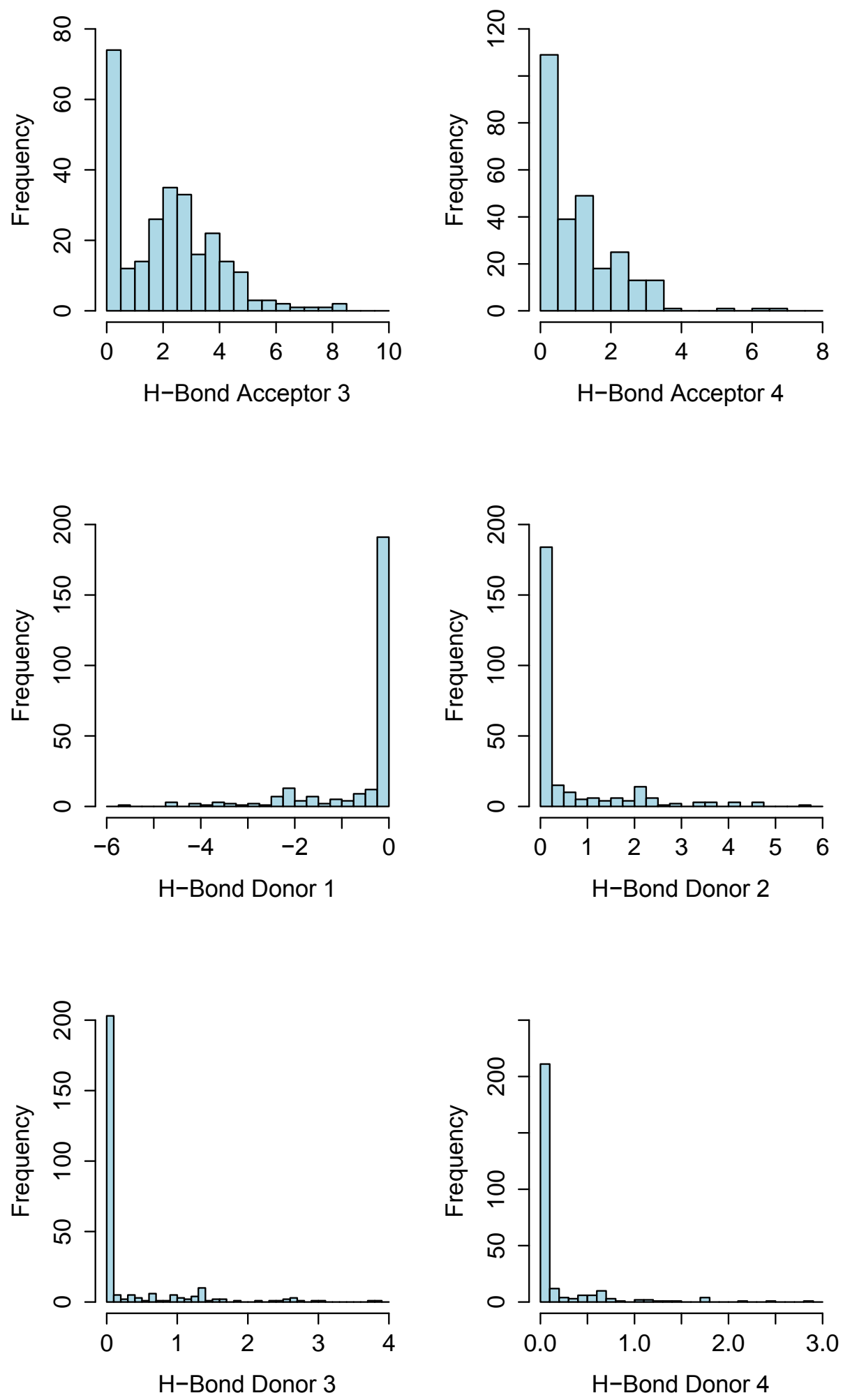

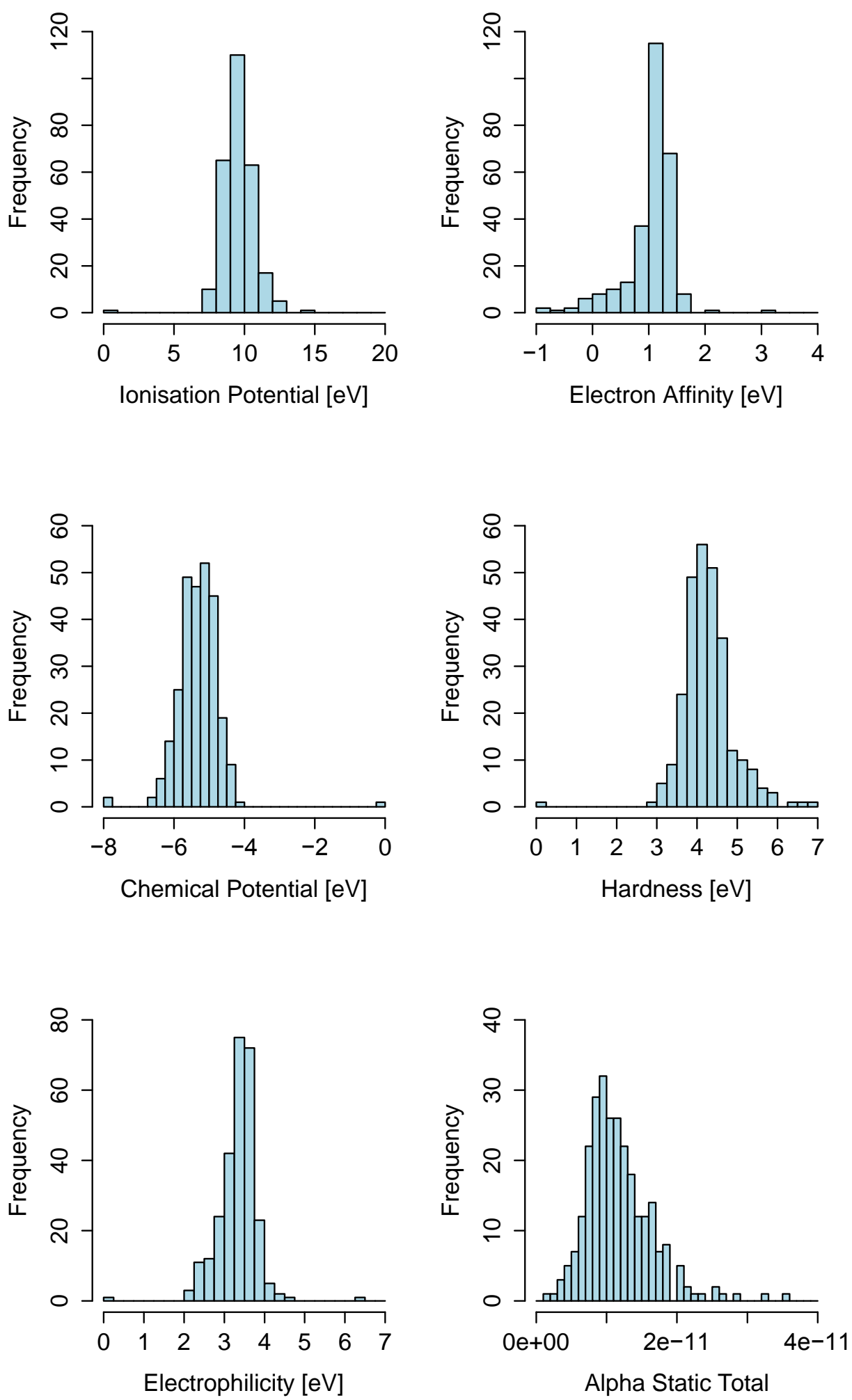

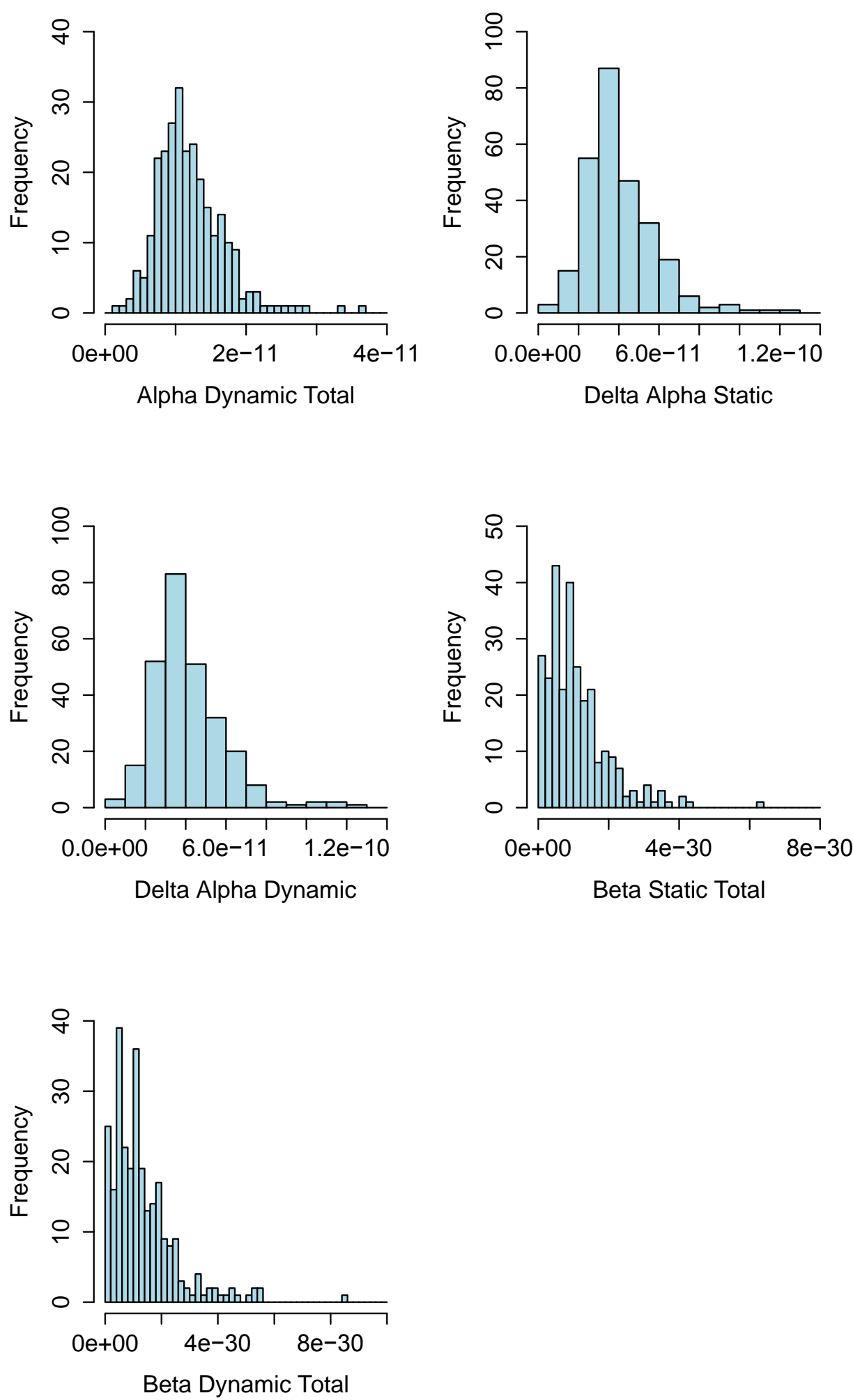


\section{Pls Physical Properties versus Computed Descriptors: Predicted - Observed Plots.}

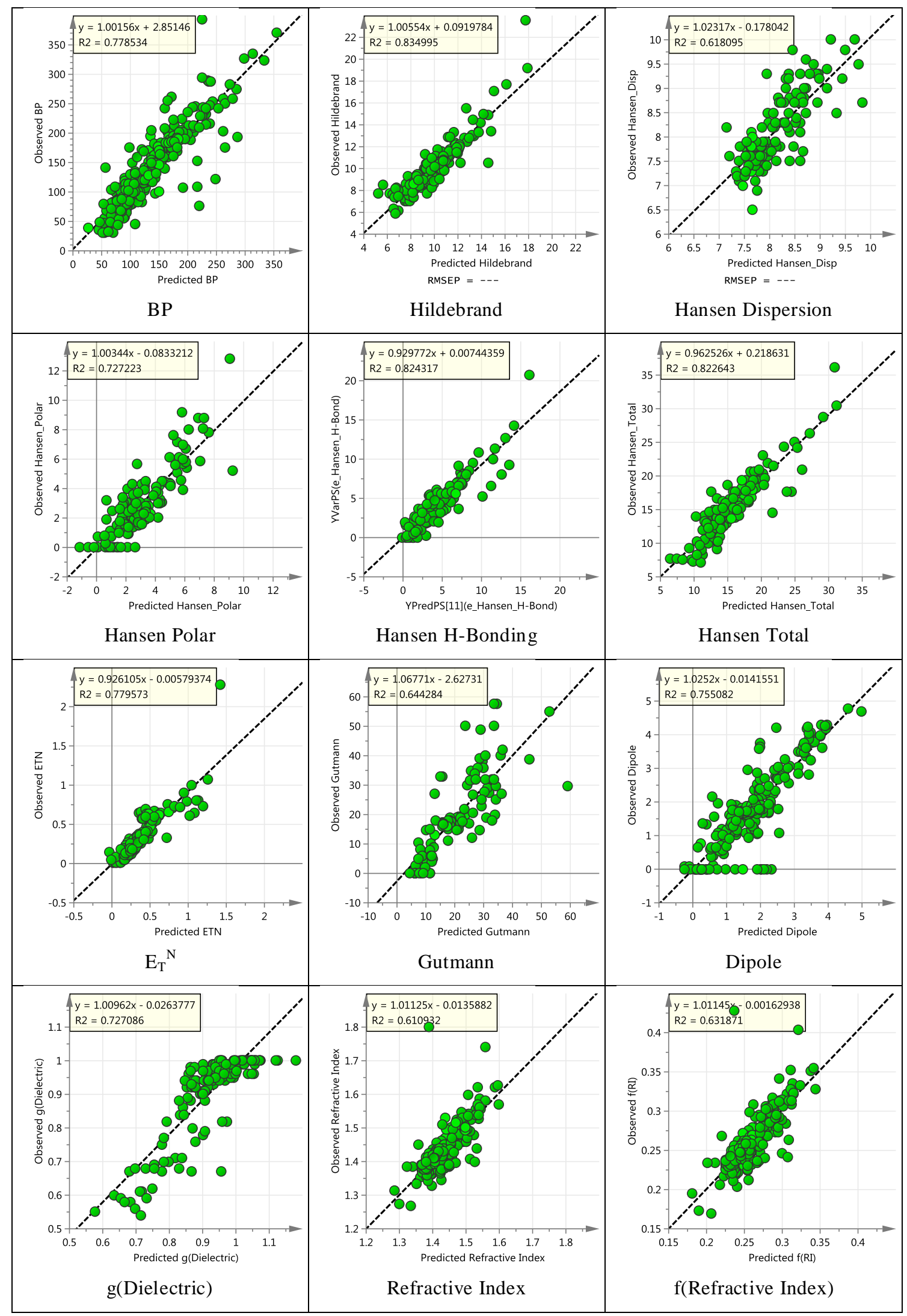




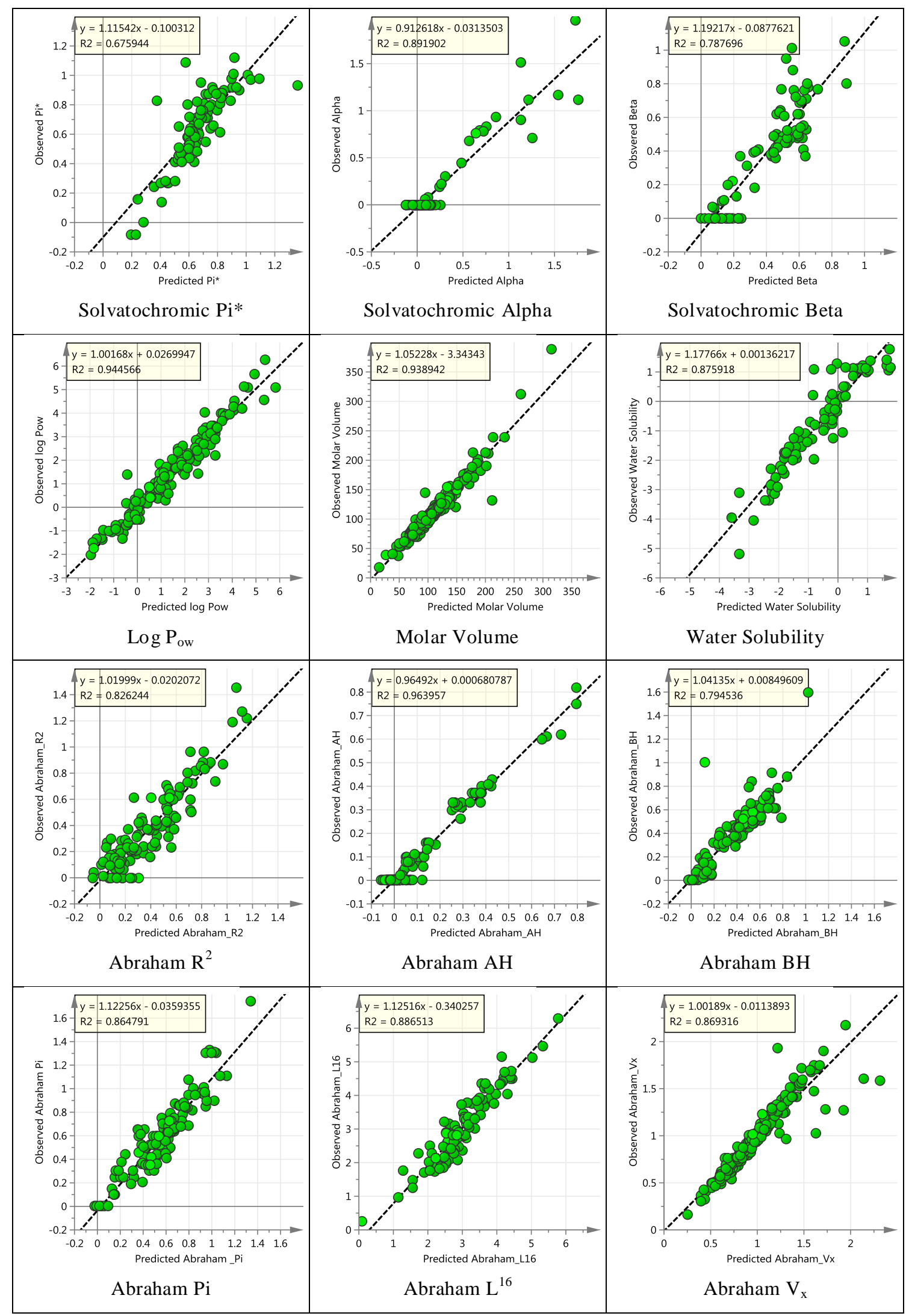




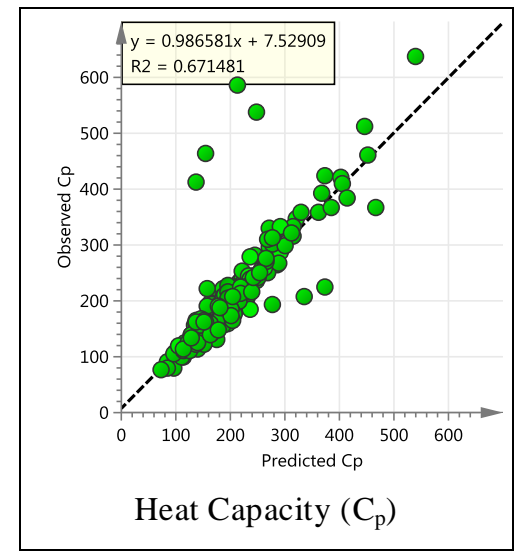

